\title{
Ecological correlates of gene family size in a pine-feeding sawfly genome and across Hymenoptera
}

Kim L. Vertacnik ${ }^{1,2}$, Danielle K. Herrig ${ }^{1}$, R. Keating Godfrey ${ }^{3}$, Tom Hill ${ }^{4,5}$, Scott M. Geib ${ }^{6}$, Robert L. Unckless $^{4}$, David R. Nelson ${ }^{7}$, and Catherine R. Linnen ${ }^{1}$

${ }^{1}$ Department of Biology, University of Kentucky, Lexington, KY 40506, USA

${ }^{2}$ Columbia River Inter-Tribal Fish Commission, Hagerman, ID 83332, USA (current address)

${ }^{3}$ Department of Neuroscience, University of Arizona, Tucson, AZ 85721, USA

${ }^{4}$ Department of Molecular Biosciences, University of Kansas, Lawrence, Kansas 66045, USA

${ }^{5}$ NIH Frederick National Laboratory for Cancer Research, Frederick, MD 21702, USA

${ }^{6}$ Tropical Crop and Commodity Protection Research Unit, United States Department of Agriculture: Agriculture Research Service Pacific Basin Agricultural Research Center, Hilo, Hawaii 96720, USA ${ }^{7}$ Department of Microbiology, Immunology and Biochemistry, University of Tennessee Health Science Center, Memphis, TN 38163, USA

Correspondence: kvertacnik@critfc.org

Keywords: evolutionary predictability, adaptation, chemosensation, detoxification, immunity,

21 Hymenoptera, draft genome 


\section{Abstract}

A central goal in evolutionary biology is to determine the predictability of adaptive genetic

24 changes. Despite many documented cases of convergent evolution at individual loci, little is known about

25 the repeatability of gene family expansions and contractions. To address this void, we examined gene

26 family evolution in the redheaded pine sawfly Neodiprion lecontei, a non-eusocial hymenopteran and

27 exemplar of a pine-specialized lineage evolved from angiosperm-feeding ancestors. After assembling and

28 annotating a draft genome, we manually annotated multiple gene families with chemosensory,

29 detoxification, or immunity functions and characterized their genomic distributions and evolutionary

30 history. Our results suggest that expansions of bitter gustatory receptor (GR), clan 3 cytochrome P450

31 (CYP3), and antimicrobial peptide (AMP) subfamilies may have contributed to pine adaptation. By

32 contrast, there was no evidence of recent gene family contraction via pseudogenization. Next, we

33 compared the number of genes in these same families across insect taxa that vary in diet, dietary

34 specialization, and social behavior. In Hymenoptera, herbivory was associated with small GR and

35 olfactory receptor (OR) families, eusociality was associated with large OR and small AMP families,

36 and - unlike investigations in more closely related taxa-ecological specialization was not related to gene

37 family size. Overall, our results suggest that gene families that mediate ecological interactions may

38 expand and contract predictably in response to particular selection pressures, however, the ecological

39 drivers and temporal pace of gene gain and loss likely varies considerably across gene families.

\section{Introduction}

Changes in gene family size are a potentially important source of evolutionary innovation. When gene families grow via duplication, for example, reduced functional constraints may facilitate the development of phenotypic novelty (Ohno 1970; Demuth and Hahn 2009). Reductions in gene family size can also enable novel traits. For example, the colonization of highly specialized niches like oligotrophic caves (Protas et al. 2006; Gross et al. 2009; Yang et al. 2016) and toxic host plants (Matsuo et al. 2007;

47 McBride 2007; Good et al. 2014) is linked to rampant pseudogenization. Together, these observations

48 suggest that gene families predictably expand or contract in response to specific selection pressures. Yet

49 compared to the rich and growing literature on genetic convergence at individual loci (Martin and

50 Orgogozo 2013), the repeatability and predictability of gene family evolution remains understudied.

51 The evolution of many gene families, defined here as groups of genes that share sequence and

52 functional similarity from common ancestry (Dayhoff 1976; Demuth and Hahn 2009), is consistent with a

53 birth-death model where genes arise via duplication (gene gain) and are lost via deletion or

54 pseudogenization (gene loss) (Hughes and Nei 1992; Nei and Rooney 2005). When frequency rates of 
duplication and deletion evolve primarily through genetic drift, over time gene family sizes contract and expand via a process dubbed genomic drift (Nei 2007; Nozawa et al. 2007). Overall, the stochastic birthdeath process of genomic drift (which differs from Nei's conceptual birth-death model of gene family evolution (Hahn et al. 2005)) sufficiently explains most gene family size distributions within genomes (Karev et al. 2002) and between species (Hahn et al. 2007).

But during an ecological shift, natural selection can influence birth-death dynamics by promoting the expansion or contraction of specific gene families. Thus, taxa adapted to a novel niche may have genomic evidence of selective maintenance for gene duplications or deletions. For example, if selection favors gene gain, novel gene duplicates will tend to persist in the population and form subfamilies of recently diverged paralogs. If the mutational mechanism that generates new duplicate genes is unequal crossing over during meiosis, these recently diverged paralogs will be arranged in tandem arrays across the genome (Zhang 2003). Moreover, if duplicates experience positive selection for novel functions, they can have elevated amino acid substitution rates. Conversely, some genetic functions may become obsolete or even deleterious in the novel habitat. In this case, positive or relaxed purifying selection will cause some gene families to accumulate loss-of-function mutations at an accelerated rate.

After an ecological shift, impacted gene families will eventually reach a new equilibrium state where gene number evolves primarily through negative selection and genomic drift. Likewise, tandem array lengths will reflect local recombination rates (Akhunov et al. 2003; Zhang and Gaut 2003; Rizzon et al. 2006; Thomas 2006) and pseudogenes will fade into the genomic background (Petrov et al. 1996;

74 Petrov and Hartl 1997, 1998). Thus, within-genome signatures of adaptive changes in gene family size are 75 likely ephemeral and best detected in lineages that recently shifted to a novel niche. Plus, if selection consistently favors the expansion or contraction of specific gene families in specific environments, among-taxon correlations between gene family size and ecology should be maintained. Currently, the extent to which different taxa converge at the level of gene family size changes is largely unknown.

Arguably, the genes most likely to expand and contract convergently in response to similar selection pressures are those that mediate organismal interactions with their biotic and abiotic environments. These "environmentally responsive genes" include chemosensory (e.g., olfactory and gustatory receptors), detoxifying (e.g., cytochrome P450), and immunity (e.g., immunoglobulin and

83 MHC) genes. To cope with constantly changing pressures, environmentally responsive genes tend to be 84 characterized by elevated sequence diversity, duplication rates, substitution rates, and genomic clustering, 85 as well as tissue- or temporal-specific expression (Berenbaum 2002) and limited pleiotropy (Arguello et 86 al. 2016). Importantly, causal links between changes in environmentally responsive genes and adaptation 87 to novel niches have been established for multiple taxa (Després et al. 2007; Matsuo et al. 2007; Dobler et 
With exceptionally diverse ecologies and an ever-increasing availability of annotated genomes (Consortium 2013; Poelchau et al. 2015), insects are a powerful system for investigating the predictability of size changes in environmentally responsive gene families. To date, at least two ecological transitions are hypothesized to have a predictable impact on gene family size in insect lineages. In plant-feeding insects, the evolution of increased dietary specialization (i.e., smaller diet breadth) is associated with reduced chemosensory and detoxifying gene family sizes (McBride 2007; McBride and Arguello 2007;

95 Good et al. 2014; Goldman-Huertas et al. 2015; Calla et al. 2017; Comeault et al. 2017) but see (Gardiner

96 et al. 2008). In hymenopteran insects, eusociality is associated with expansions of the olfactory-receptor

97 family and contractions of the gustatory-receptor family (Robertson and Wanner 2006; Zhou et al. 2015;

98 McKenzie et al. 2016; Brand and Ramírez 2017) but see (Fischman et al. 2011; Johnson et al. 2018).

99 Most of these studies, however, consider a single ecological characteristic or gene family (but see

100 Robertson and Wanner 2006) which is problematic since changes in social behavior may often be

101 accompanied by changes in other ecological characteristics and vice versa (Faulkes et al. 1997; Duffy and

102 Macdonald 2010; Ross et al. 2013). A better understanding of ecology and gene family size relationships

103 requires simultaneous consideration of multiple ecological characteristics and diverse gene families.

Here, we characterize multiple environmentally responsive gene families in the genome of the redheaded pine sawfly, Neodiprion lecontei (Order: Hymenoptera; Family: Diprionidae). This species provides an opportunity to examine both within-genome signatures of adaptive gene family contractions

107 and expansions, and among-lineage correlations between ecology and gene family size. First, for within-

108 genome signatures, $N$. lecontei is an exemplar of an herbivorous hymenopteran lineage (Diprionidae) that

109 underwent a drastic host shift: sometime within the last 60 million years, this lineage transitioned from

110 angiosperms to coniferous host plants in the family Pinaceae (Boevé et al. 2013; Peters et al. 2017). To

111 defend against herbivores and pathogens, Pinaceae produce viscous oleoresin secretions that are sticky

112 and have unique antimicrobial properties (Trapp and Croteau 2001; Gershenzon and Dudareva 2007). To

113 manage these toxic and extraordinarily sticky resins, $N$. lecontei and related diprionids evolved

114 specialized feeding and egg-laying traits (Figure 1). Beyond these traits, we hypothesize that pine

115 specialization likely resulted in pronounced changes to the selection pressures acting on multiple gene

116 families, especially those involved in chemosensation, detoxification, and immune function. Second, with

117 respect to among-lineage correlations between ecology and gene-family size, $N$. lecontei is an

118 herbivorous, non-eusocial insect from the Eusymphyta, a massively understudied hymenopteran clade

119 (Peters et al. 2017). Although many assembled and annotated hymenopteran genomes are currently

120 available, almost all have come from apocritans (bees, wasps, and ants, but see (Robertson et al. 2018)).

121 Thus, $N$. lecontei increases the ecological, behavioral, and taxonomic diversity of hymenopteran genomes

122 for evaluating ecological correlates of gene family size among taxa. 
To evaluate the predictability of gene family evolution, we assembled a draft genome for $N$.

124 lecontei and manually annotated genes for five environmentally responsive gene families: olfactory

125 receptor (OR), gustatory receptor (GR), odorant binding protein (OBP), cytochrome P450 (CYP), and

126 antimicrobial peptide (AMP). For gene families that underwent a size change related to pine adaptation,

127 we expected one or more of the following patterns: (1) clusters of recently diverged paralogs in gene-

128 family trees, (2) a high proportion of genes in tandem arrays, (3) signatures of positive selection among

129 paralogs, and (4) elevated rates of pseudogenization. Then, for the same five gene families, we asked

130 whether gene-family size correlated with ecology among distantly related insect taxa. To do so, we

131 compiled published gene annotations and ecological variables (diet type, degree of ecological

132 specialization, presence/absence of eusociality) for hymenopteran taxa. Together, these analyses identify

133 possible candidate gene families underlying pine specialization and reveal that relationships between gene

134 family size and ecology differ among environmentally responsive gene families.

\section{Results}

137 Genome assembly and annotation

138 Sequencing and assembly

139 We sequenced one mate-pair and two small-insert Illumina libraries made from haploid male

140 siblings (see Methods). After read processing, we retained 268 billion PE100 reads with a combined read

141 depth of 112x (Table S1). ALLPATHS-LG (v47417) (Gnerre et al. 2011) produced a 239-Mbp assembly

142 consisting of 4523 scaffolds, with a scaffold N50 of $243 \mathrm{kbp}$ (Table S2). Prior studies identified seven

143 chromosomes in N. lecontei (Smith 1941; Maxwell 1958; Sohi and Ennis 1981; Linnen et al. 2018). With

144 an estimated genome size (1C) of $331 \pm 9.6 \mathrm{Mbp}$, our assembly captured $72 \%$ of the genome. Overall,

145 these metrics are comparable to other hymenopteran assemblies (Table S2).

146 To measure assembly completeness and artificial sequence duplication, we used CEGMA (Parra

147 et al. 2007) and BUSCO (Simão et al. 2015). Both search the assembly for a set of single-copy, conserved

148 genes, however, the CEGMA software has been deprecated (http://korflab.ucdavis.edu/Datasets/cegma).

149 Of the 248 CEGMA core eukaryotic genes, $90 \%$ aligned as complete, single copies and $8 \%$ aligned

150 complete but duplicated. For BUSCO, we used the OrthoDB arthropod dataset, and out of 2675 groups

$15177 \%$ were complete, single copies and $3 \%$ were complete but duplicated. These metrics indicate the

152 presence of artificial duplicate sequences, but otherwise the assembly was reasonably complete and 153 suitable for annotation.

154 About $15.8 \%$ of the assembly consisted of repetitive elements, including 122 unknown

155 transposable elements that were mostly unique to $N$. lecontei (Table S3), and 212 other families of 156 transposable elements and simple repeats. This $15.8 \%$ corresponds to $11.4 \%$ of the actual $331-\mathrm{Mb}$ 
157 genome, of which we predict $27.6 \%$ is repetitive, suggesting that $\sim 16.1 \%$ of the missing $\sim 28 \%$ of the

158 genome is repetitive content (Table S3). For de novo gene prediction, we included the $N$.

159 lecontei transcriptome and protein-coding genes from Atta cephalotes (OGSv1.2), Acromyrmex echinatior

160 (OGSv3.8), Apis melifera (OGSv3.2), Athalia rosae (OGSv1.0), and Nasonia vitripennis (OGSv1.0) to

161 guide annotation. The official gene set (OGSv1) had 12,980 gene models while the transcriptome had an

162 average of 26,000 transcripts per tissue (Table S4).

163 Olfactory receptor

164 The OR gene family had 56 genes total, including the co-receptor Orco; one gene contained stop

165 codons, three were partial annotations, and 52 genes were intact (Table 1). In D. melanogaster most

166 olfactory sensory neurons (OSNs) express a single OR (along with the coreceptor, Orco), and OSNs

167 expressing a particular OR converge on a single glomerulus in the antennal lobe (Gao et al. 2000;

168 Vosshall et al. 2000; Couto et al. 2005) but see (Fishilevich and Vosshall 2005). This anatomy results in a

169 general one-to-one correspondence between the number of ORs and the number of glomeruli, a correspondence also observed in the hymenopteran European honey bee (Apis mellifera, (Robertson and

171 Wanner 2006)). Based on these studies and examination of the antennal lobes of adult male and adult

172 female $N$. lecontei, we expected to find a minimum of 49 functional ORs (Table S5, Figure 2). The close correspondence between our gene annotations and glomeruli counts suggests that we have located all or most N. lecontei OR genes.

$59 \%$ of ORs were in genomic clusters of two or more genes (Figure 3), a low proportion Neodiprion, six other hymenopterans, and D. melanogaster identified three Neodiprion-specific clades with at least five genes (Figure S1a). These same three clades were also recovered in a phylogenetic analysis of Neodiprion OR cDNA sequences (Figure S1b). For each Neodiprion-specific OR clade (and Neodiprion-specific clades in other gene families, see below), we used the Neodiprion cDNA tree, the

182 codeml program in the PAML package (Yang 2007), and likelihood-ratio tests to ask: (1) whether the 183 ratio of non-synonymous to synonymous substitution rates $(\mathrm{dN} / \mathrm{dS}$ or $\omega)$ for the focal OR clade differed

184 from the rest of the Neodiprion OR gene family and, if so, whether they exhibited evidence of positive 185 selection $(\omega>1)$ (branch tests); and (2) whether $\omega$ differed among sites across members of Neodiprion186 specific clades and, if so, which sites exhibited evidence of positive selection (site tests). For only one OR 187 clade (OR clade 1) did we detect evidence of branch-specific positive selection (i.e., rejection of both 1188 ratio and fixed- $\omega$ models), but this clade lacked evidence of site-specific positive selection (Table 2). 
The GR gene family had 44 genes total; two genes contained stop codons, two were partial annotations (one annotation was both partial and pseudogenized), and 41 were intact (Table 1). $76 \%$ of the GRs that could be placed on chromosomes were in genomic clusters (Figure 3) with three Neodiprionspecific clades of at least five genes (Figures S2a and S2b). Only one clade (GR clade 3) had evidence of

194 branch-specific positive selection (Table 2). This clade also had evidence of positive selection at some

195 amino acid positions among paralogs (Table 2; sites with evidence of positive selection include: 77E,

$19679 \mathrm{~S}, 146 \mathrm{~N}, 275 \mathrm{~S}, 301 \mathrm{~S})$. Notably, GR Clade 3 is an expansion of six paralogs orthologous to DmGR66a,

197 a bitter receptor specifically for caffeine (Moon et al. 2006). However, N. lecontei orthologs were not

198 found for DmGR93a (Lee et al. 2009) and DmGr33a (Moon et al. 2009), coreceptors possibly required

199 for caffeine detection. Together, these data suggest that caffeine-like GR receptors have been coopted for 200 novel functions in N. lecontei.

The GR family also had orthologs for sugar receptors DmGR5a (trehalose) (Dahanukar et al. 2001), DmGR43a (fructose) (Miyamoto et al. 2012), and DmGR64a-f (multiple sugars) (Slone et al.

204 Orthologs to these carbon dioxide receptors have not been found in Apocrita but seem to be preserved in 205 Symphyta, like N. lecontei (Robertson and Kent 2009; Robertson et al. 2018).

\section{Odorant binding protein}

207 The OBP gene family had 13 genes total; none were pseudogenized or partial annotations (Table

208 1). In this family, $38 \%$ of genes were in genomic clusters, including a cluster of five genes on

209 chromosome 6 (Figure 3). Neodiprion-specific OBP clades were not found, even for the chromosome 6

210 cluster. We note, however, that the OBP phylogenies had low bootstrap support (Figure S3a,b), making it

211 difficult to infer relationships among paralogs.

212 Cytochrome P450

213 The CYP gene family had 107 genes total; twelve genes contained stop codons, two were partial

214 annotations, and 93 were intact (Table 1). In insects, CYPs belong to four major clades, which are

215 referred to as clans (Feyereisen 2012). When we split the CYP gene family by clan, the CYP2 clan had

216 nine intact genes; the CYP3 clan had 47 intact genes and eight pseudogenes; the CYP4 clan had 27 intact

217 genes, four pseudogenes, and two partial genes; and the mitochondrial CYP clan had 10 intact genes

218 (Table 1). Across all CYPs, 66\% were in genomic clusters (Figure 3). Looking at the four major clans

219 separately, the percentage of clustered genes were: 33\% for CYP2, 81\% for CYP3, 55\% for CYP4, and

$22050 \%$ for mitochondrial CYP.

221 The CYP gene family had five Neodiprion-specific clades with at least five genes (Figure S4a,b),

222 four of which were in the CYP3 clan. Of these, two clades that were both within the CYP3 clan (CYP

223 clades 3 and 5) had evidence of branch-specific, but not site-specific, positive selection (Table 2). CYP 
224 clade 3 contained members of the CYP6 subfamily, and the CYP clade 5 contained members of the

225 CYP336 subfamily. Several studies to date suggest that members of the CYP3 clan-and the CYP6

226 subfamily in particular-play an important role in detoxifying pesticides and host-plant allelochemicals

227 (Feyereisen 2012).

Orthologs were found for all the Halloween genes (which include genes from both the CYP2 and mitochondrial CYP clans) of the 20-hydroxy ecdysone biosynthesis pathway: CYP302A1 (disembodied),

230 CYP306A1 (phantom), CYP307A2 (spookier), CYP307B1 (spookiest), CYP314A1 (shade), CYP315A1

231 (shadow), and CYP18A1 which turns over 20-hydroxy ecdysone (Rewitz et al. 2007; Feyereisen 2011;

232 Guittard et al. 2011; Qu et al. 2015). The juvenile hormone biosynthesis gene CYP15A1 was present as

233 well (Helvig et al. 2004). Finally, N. lecontei had orthologs for the two CYP4G enzymes that synthesize

234 the cuticular hydrocarbons used as external waterproof coating (Qiu et al. 2012).

235 Immunity

Antimicrobial peptides (AMPs) are expressed upon infection to kill or inhibit microbes. Based on hymenopteran sequences, the $N$. lecontei AMP gene family had 21 genes (Table 1; Table S6), including single copies of Hymenoptaecin, Abaecin, and Tachystatin, but no clear Defensin ortholog. Over 18 Hisnavicin genes were identified, including a Neodiprion-specific expansion of eight histidine-rich paralogs orthologous to Hisnavicin-4, which has been characterized as a larval cuticle protein and AMP, but not functionally tested (Tian et al. 2010). The N. lecontei Hisnavicins had a conserved 62 amino acid motif that appeared up to 19 times in a single protein; the purpose of this amplification is unknown. $95 \%$ of the AMPs were in genomic clusters (Figure 3). Due to low bootstrap support on many of the branches

244 in our Hisnavicin protein tree, we could not identify unambiguous Neodiprion-specific clades (Figure S5a). However, our Neodiprion cDNA tree (Figure S5b) did reveal strong support for the monophyly of a cluster of 15 Hisnavicins on linkage group 5 (Figure 3), and this cluster had some evidence of positive selection (Table 2).

Outside of the AMP family, most immune pathways had direct orthologs between N. lecontei and D. melanogaster (Figure S6, Table S7). The basic viral siRNA response pathway was completely conserved between species. The immune deficiency (IMD) pathway was missing an ortholog for the peptidoglycan recognition receptor $P G R P-L C$, but it is likely that another $P G R P$ replaced $P G R P-L C$ in $N$.

252 lecontei; assigning PGRP orthology was also difficult in ants (Gupta et al. 2015). Also missing is the 253 Drosophila mitogen activated protein kinase kinase kinase, TGF- $\beta$ activated kinase 1 (Takl), but $N$. 254 lecontei had a similar TGF- $\beta$ activated kinase that is a close ortholog to several Tak1-like D. melanogaster proteins possibly involved in immune deficiency signaling. The encapsulation/melanization pathway was missing one of the two Drosophila GTPases (Rak2). The N. lecontei Rak1 ortholog may be 
Duox pathway was missing the top G-protein coupled receptor, but this is unknown in D. melanogaster and unidentified in other Hymenoptera (Evans et al. 2006). Interestingly, N. lecontei had two copies of Dual Oxidase (Duox), which regulates commensal gut microbiota and infectious microbes (Ha et al. 2005; Lee et al. 2015); Apis mellifera had one copy. Finally, the Toll pathway NF-kappaB transcription factor, Dorsal-related immunity factor (Dif) does not have a one-to-one ortholog in N. lecontei, but two copies of 263 its paralog, Dorsal, were present.

\section{Within-genome signatures of adaptive expansions and contractions}

265 Evidence of selection in Neodiprion-specific gene family clades

Massive gene family expansions with dozens of genes were not found in $N$. lecontei (in contrast to (Smadja et al. 2009; Zhou et al. 2015)). Instead, the largest Neodiprion-specific clade had 22 genes (CYP gene family) and the rest had fewer than 10 genes. Nevertheless, we did identify 11 Neodiprionspecific clades containing at least 5 closely related paralogs and a monophyletic clade of 15 AMPs with ambiguous ancestry (Table 1). Of these 12 clades, four had significant branch positive selection (OR clade 1, GR clade 3, CYP clade 3, and CYP clade 5) (Table 2). Of these four clades, only one also had significant site-specific positive selection (GR clade 3) (Table 2).

\section{Clustering}

Our five focal gene families varied in the proportion of genes that were found in clusters of two or more genes (Fisher's exact test, $P=0.002$; Table 1). Post-hoc tests revealed that much of this variation was due to differences between the highly clustered AMP family and all other families except GR (AMP vs. OR: $P=0.0091$; AMP vs. OBP: $P=0.0053$; AMP vs. CYP: $P=0.024$, AMP vs. GR: $P=0.12$; all pvalues are FDR-corrected). The only other difference in clustering that we detected was between the GR and singleton-heavy OBP families (FDR-corrected $P=0.045)$.

Differences in clustering were even more pronounced when we separated the CYP family by clan (Fisher's exact test, $P<0.0001$; Table 1). In addition to the pairwise differences described above, we also found that the proportion of CYP3 genes found in clusters differed significantly from ORs, CYP2s, and CYP4s (all FDR-corrected $P<0.05$ ), but not AMPs, GRs, and mitochondrial CYPs. Additionally, AMP clustering differed from CYP2, CYP4, and mitochondrial CYP, while GR differed from CYP2 (all FDRcorrected $P<0.05$ ). Together, these analyses identified AMP, GR, and CYP3 as having an unusually high proportion of genes found in clusters compared to other environmentally responsive gene families. Pseudogenization

Overall, we found very few pseudogenes, and the proportion of pseudogenized genes did not differ significantly among gene families (Fisher's exact test, $P=0.12$; Table 1 ). The chemoreceptors had one pseudogene each while CYP had 12, which is about $10 \%$ of the family, but this was also the largest gene family. Although CYP3 had more pseudogenes than other CYP clans, the proportion of 
292 pseudogenized genes still did not differ when we compared CYP clans (Fisher's exact test, $P=0.10$ ).

293 Given these low rates of pseudogenization, it is unlikely that $N$. lecontei gene families underwent

294 substantial, recent contractions.

295 Ecological correlates of gene-family size across insects

We first examined broad-scale variation in the sizes of our five focal gene families and four CYP clans among different insect orders (Figure S7). Not surprisingly, sample sizes were highly variable across gene families and insect orders. Despite this variation, we observed some intriguing differences among gene families and taxa. We detected significant differences in gene family size among orders for OR (Kruskal-Wallas chisq $\left.=48.2, \mathrm{df}=12, P<1 \times 10^{-5}\right), \mathrm{GR}(\mathrm{K}-\mathrm{W}$ chisq $=25.5, \mathrm{df}=9, P=0.0025)$, and OBP $\left(\mathrm{K}-\mathrm{W}\right.$ chisq $\left.=37.6, \mathrm{df}=9, P<1 \times 10^{-4}\right)$, but not CYP $(\mathrm{K}-\mathrm{W}$ chisq $=10.3, \mathrm{df}=7, P=0.17)$ or AMP $(\mathrm{K}-\mathrm{W}$ chisq $=7.93, \mathrm{df}=5, P=0.16)$. We note, however, that the AMP sample size was considerably smaller than the other gene families. When we looked at CYP clans individually, we found differences among orders for CYP4 $(\mathrm{K}-\mathrm{W}$ chisq $=19.0, \mathrm{df}=7, P=0.0083)$ and mitochondrial CYP $(\mathrm{K}-\mathrm{W}$ chisq $=16.3, \mathrm{df}=7, P=0.022)$, but not CYP2 $(\mathrm{K}-\mathrm{W}$ chisq $=9.19, \mathrm{df}=7, P=0.24)$ or CYP3 $(\mathrm{K}-\mathrm{W}$ chisq $=8.76, \mathrm{df}=7, P=0.27)$.

For the OR family, among-group differences in gene number were mostly attributable to an unusually large number of OR genes in Hymenoptera (significant post-hoc tests include Diptera vs. Hymenoptera: $P=0.0018$; Hemiptera vs. Hymenoptera: $P=0.00014$; and Odonata vs. Hymenoptera: $P=$ 0.011; all p-values are FDR-corrected). By contrast, the size of the OBP family was larger in Diptera than other orders (significant post-hoc tests include Diptera vs. Hymenoptera: $P=0.00037$; Diptera vs. Hemiptera: $P=0.00092$; all p-values are FDR-corrected). Although none of the post-hoc tests were

313 significant for GR family size, the Blattodea appear to have more GRs on average than other insect orders 314 (Figure S7). For CYP clans, posthoc tests revealed that hymenopterans have fewer CYP4s than dipterans 315 (FDR-corrected $P=0.010$ ) and fewer mitochondrial CYPs than both dipterans and lepidopterans (FDR316 corrected $P=0.024$ and 0.023 , respectively).

317 We next examined how gene family size correlated with ecology within the hymenopteran clade 318 (Figures 4 and 5). Once again, we observed differences among gene families. We found that the number 319 of ORs differed significantly among hymenopteran species that differed in diet (Kruskal-Wallas chisq $=$ $32015.8, \mathrm{df}=3, P=0.0012$ ) and sociality (Wilcoxon rank-sum test $\mathrm{W}=115 ; P=0.00094$ ). For diet, we 321 found that herbivores had fewer ORs than all other diet types (fungivores vs. herbivores: $P=0.015$; 322 omnivores vs. herbivores: $P=0.015$; insectivores vs. herbivores: $P=0.048$; all $\mathrm{p}$-values are FDR323 corrected). We observed an even more striking difference between eusocial and non-eusocial 324 hymenopterans, with the former having larger OR families, on average. By contrast, GR family size was 325 related to diet (Kruskal-Wallas chisq $=11.8, \mathrm{df}=3, P=0.0082$ ), but not sociality (Wilcoxon rank sum 
326 test $\mathrm{W}=30 ; P=0.65)$. And CYP family size was related to sociality $(\mathrm{W}=2 ; P=0.045)$, but not $\operatorname{diet}(P=$

327 0.38). Finally, specialists and generalists did not differ significantly in gene family size in any of the gene

328 families and ecology was unrelated to gene family size for OBP and CYP (total CYP number and

329 individual CYP clans). Although these analyses have several limitations (see discussion), these results are

330 consistent with the hypothesis that environmentally responsive gene families may contract or expand

331 predictably in response to particular selection pressures.

\section{Discussion}

The predictability of gene family expansion or contraction in response to specific selection pressures is still an open question. Here, we evaluated genomic signatures of adaptive gene family size

336 changes in five environmentally responsive gene families within the $N$. lecontei draft genome, a

337 hymenopteran exemplar of a pine-specialized lineage. Although we saw minimal evidence of recent gene

338 loss via pseudogenization, at least three gene families (AMP, GR, and CYP3) had genomic distributions

339 consistent with the selective maintenance of novel gene duplicates, and two of these families also had

340 evidence of positive selection within Neodiprion-specific clades (GR and CYP3). Next, we examined

341 these same gene families in other hymenopterans to see if family size correlated with diet, ecological

342 specialization, or eusocial behavior. Among Hymenoptera, we found that OR family size was correlated

343 with eusociality and diet type, but not dietary specialization; GR family size was correlated with diet type;

344 and AMP family size was associated with eusociality. These results suggest that ecology can have a

345 predictable impact on gene family size and that different selection pressures impact different gene

346 families. Below, we discuss both the implications and limitations of our analyses and suggest priorities

347 for future comparative work on gene family size evolution.

\section{Within-genome signatures of gene-family size change}

349 During a niche shift, new selective pressures can leave footprints in the genomes of evolving

350 lineages; such signatures of positive selection are well described for individual loci (Nielsen et al. 2005;

351 Vitti et al. 2013). Similarly, strong selection for increases or decreases in the size of a particular gene

352 family should also leave characteristic genomic footprints. We argue that these footprints include

353 monophyletic groups of closely related paralogs in gene-family trees (from the selective maintenance of

354 novel duplicates), genomic clustering (when novel genes arise via unequal crossing over), evidence of

355 positive selection among paralogs (given selection for sub- or neofunctionalization), and high rates of

356 pseudogenization (from the selective maintenance of loss-of-function mutations). Of the environmentally

357 responsive gene families we evaluated, none exhibited patterns consistent with selection for a decrease in

358 gene family size. By contrast, at least three families had characteristics consistent with selection for an

359 increase in gene family size. Two of these families, GR and CYP3, were highly clustered in the genome 
and exhibited evidence of positive selection, making these especially promising candidates for expansions related to a novel coniferous host. Additionally, although the AMP family lacked evidence of positive selection, its unusually clustered distribution in the Neodiprion genome could be related to selection for increased dosage of a conserved protein function (Perry et al. 2007). Below we discuss the functions of these three candidate families in more detail.

Shifts to pine feeding likely involved changes in the detection of and response to pine-specific cues. Intriguingly, the one GR clade with evidence of positive selection - GR clade 3 - is an expansion of six paralogs (one is pseudogenized) orthologous to DmGR66a, a bitter receptor specifically for caffeine (Moon et al. 2006). However, orthologs were not found for DmGR93a (Lee et al. 2009) and DmGr33a (Moon et al. 2009), coreceptors possibly required for caffeine detection. Nevertheless, honeybees, which also lack clear orthologs to these putative coreceptors (Wanner and Robertson 2008), can detect and even prefer low concentrations of caffeine and nicotine (Singaravelan et al. 2005, but see de Brito Sanchez 2011). Although pines do not contain caffeine, they do synthesize alkaloids that could confer some bitterness (Mumm and Hilker 2006). Thus, despite lacking caffeine coreceptor orthologs, members of GR clade 3 may still be involved in the detection of pine-specific bitter compounds. Duplications of putative bitter GRs are documented in other host-specialized insects, such as Heliconius, Danaus, and Bombyx butterflies (Wanner and Robertson 2008; Briscoe et al. 2013). Our sawfly-specific GR expansion, coupled with the finding that GR family size is associated with diet (see below), lends support to the hypothesis that expansions of GR bitter receptors repeatedly contribute to changes in oviposition and feeding behaviors in plant-feeding insects.

Because pines contain toxic components like terpenoids and phenolics, detoxifying gene families are also promising candidates for pine adaptation. The mountain pine beetle (Dendroctonus ponderosae), feeds on pine bark and wood and has gene "blooms" (species-specific gene gains) in the CYP3 and CYP4 clans (Keeling et al. 2013). Similarly, in N. lecontei, the CYP family had five blooms (Figure S4a): four CYP3 and one CYP4. CYP3 blooms are also found in wood-feeding insects that do not use pine, such as the emerald ash borer (Agrilus planipennis) (David Nelson, unpublished data) and the Asian longhorned beetle (Anoplophora glabripennis) (McKenna et al. 2016). Notably, N. lecontei larvae frequently ingest pine bark in addition to pine needles (Wilson 1992), suggesting that CYP3 may expand predictably in wood feeders. Additionally, one of the two Neodiprion-specific CYP3 clades with evidence of positive selection (Table 3) belongs to the CYP6 subfamily, which is linked to host plant adaptation in several insect taxa (Li et al. 2003; Li et al. 2007; Feyereisen 2012; Mittapelly et al. 2019).

Because pine resin has antimicrobial (Himejima et al. 1992; Cowan 1999; Gershenzon and

392 Dudareva 2007) and fungicidal properties (Grayer and Harborne 1994), we hypothesized that N. lecontei

393 co-opted these compounds for its own defense, leading to relaxed selection on genes involved in 
394 immunity and a reduced innate immune response. In other Hymenoptera, honeybees (Apis mellifera)

395 exposed to plant resin have reduced expression of immune-related genes (Simone et al. 2009) and wood

396 ants (Formica paralugubris) that use conifer resin as building material have slightly reduced inducible

397 immune system activity and nests with lower bacterial and fungal loads (Castella et al. 2008). In Diptera,

398 AMP loss is associated with herbivorous lineages that live within host tissue, a more sterile habitat than is

399 experienced by most dipterans (Hanson et al. 2019). Unexpectedly, we found a large species-specific

400 clade of Hisnavicin-like AMPs in Neodiprion. Although additional data are needed to confirm that

401 Hisnavicin orthologs act as AMPs in N. lecontei, one possible explanation for this putatively adaptive

402 expansion that lacked an accompanying change in non-synonymous substitution rate is that having large

403 numbers of Hisnavicin-like AMPs confers protection against pathogens unique to pine trees. That said,

404 our data do not rule out adaptive AMP loss. For example, N. lecontei lacks a clear Defensin ortholog, a

405 gene present in all dipterans tested to date (Hanson et al. 2019).

406 Limitations of within-genome analyses

407 One benefit to studying adaptive expansions/contractions within a single taxon is that gene

408 families have likely experienced similar demographic histories, which can also impact gene birth and

409 death rates. That said, each of our within-genome signatures of selection has limitations that should be

410 revisited with additional data. First, our analysis of genomic clustering does not account for local

411 recombination rate variation, which correlates with tandem array size in several taxa (Gaut et al. 2007). A

412 fine-scale recombination rate map, coupled with clustering analyses for many additional gene families,

413 would more rigorously test the extent to which individual gene family clustering deviates from the

414 genome-wide relationship between recombination rate and tandem array size.

415 Second, a lack of comparable data from other Eusymphyta meant that our gene family

416 phylogenies lacked orthologues from closely related sawfly taxa. Thus, the "Neodiprion-specific" clades

417 may not be unique to pine-feeding sawflies. If these paralogs were present prior to the shift to pine hosts,

418 this would not support a scenario in which new duplicates were selectively maintained in the novel niche.

419 Signatures of positive selection may still be related to pine adaptation but would indicate selection on

420 preexisting loci rather than selection favoring gene family expansion.

421 Third, signatures of adaptive gene family expansions and contractions may be ephemeral, and the

422 shift to pine use could have occurred too long ago to detect these signatures in N. lecontei. For example,

423 in Drosophila, pseudogenes have an estimated half-life of $\sim 14.3$ million years (Petrov et al. 1996; Petrov

424 and Hartl 1997, 1998). If the rate of gene decay is similar in Neodiprion, then pseudogenes that formed

425 after a shift to pine (up to 60 mya) may no longer be detectable in the genomes of extant sawflies.

426 Likewise, gene clustering patterns are likely to change over time from chromosomal rearrangements and

427 additional gene duplications and deletions. To investigate how the number and position of genes in these 
428 focal families has changed over time, high quality gene annotations for diprionids and many additional

429 sawfly outgroups are needed. Fortunately, even if footprints of recent gene family size changes are too

430 ephemeral to be detected in most taxa, consistent relationships with ecology should still be detectable

431 given sufficient sampling of taxa differing in ecological traits of interest.

\section{Ecological correlates of gene family size among hymenopteran taxa}

433 The largest insect OR gene families are in eusocial Hymenoptera, leading to the hypothesis that

434 OR family size expansions were favored in these lineages because they facilitate complex chemical

435 communication (Robertson and Wanner 2006; LeBoeuf et al. 2013; Zhou et al. 2015). To date, evidence

436 in support of this hypothesis has been mixed (e.g., (Roux et al. 2014; Brand and Ramírez 2017).

437 Consistent with the OR-eusociality hypothesis, we found that, on average, eusocial hymenopterans had

438 larger OR families than non-eusocial hymenopterans. However, it is likely that eusocial taxa differ from

439 non-eusocial taxa in many other aspects of their ecology that should also impact OR evolution. Indeed,

440 we found that herbivorous hymenopterans tended to have fewer OR genes than non-herbivores.

441 Whereas all eusocial hymenopterans had relatively large OR families, some eusocial

442 hymenopterans had relatively small GR families (Figures 4, 5; (Zhou et al. 2015)). To explain the

443 strikingly small set of GR genes in honeybee, Wanner and Robinson (2006) proposed that a stable hive

444 environment and a mutualistic relationship with flowering plants resulted in a lack of selection for GR

445 expansions. Intriguingly, our data indicate that among hymenopterans, GR family size is associated with

446 diet, but not eusociality. Like the ORs, GR gene family size tends to be smaller in herbivores than in non-

447 herbivorous taxa, regardless of social behavior. The directionality of this change, however, is unclear: do

448 shifts to plant diets favor reductions in GR families, do shifts to non-plant diets favor GR expansions, or

449 is it both? Answering this question will require characterizing GR families across many independent

450 transitions to and from herbivory, as well as polarizing directions of change (i.e., distinguishing GR gains

451 from GR losses). Fortunately, there are many such diet transitions across diverse clades of insects (Wiens

452 et al. 2015).

453 Unlike sociality and diet, ecological specialization was not associated with gene family size in

454 any of the five gene families we evaluated. This result was unexpected because specialization-associated

455 reductions in gene family size are documented in diverse taxa and multiple gene families, including the

456 families examined here (McBride 2007; Smadja et al. 2009; Cao et al. 2014; Goldman-Huertas et al.

457 2015; Suzuki et al. 2018). One explanation for the lack of association between gene family sizes and

458 specialization in our data is that our "generalist" and "specialist" categories are not meaningful across

459 diverse diets (Forister et al. 2012). Additionally, within a particular diet, the degree of specialization may

460 be highly labile, with rapid fluctuations that are not captured in our broad, order-wide comparison.

461 Indeed, previous studies that reported correlations between gene family size and ecological specialization 
focused on closely related species. Thus, to fully understand how changes in ecology shape gene family evolution, it will be necessary to evaluate ecological correlates of gene family size at multiple time scales of taxonomic divergence.

Compared to ORs and GRs, our other focal gene families had far less manual annotation data available for analysis. This may explain, in part, why we did not detect strong ecological correlates for the other gene families. It is also possible that by focusing on the sizes of entire gene families, we missed relevant signals in particular subfamilies (Hahn et al. 2007). For example, as noted above, expansions of

469 CYP3 and CYP4 subfamilies are associated with wood-feeding insects and CYP3 clan subfamilies were also linked to detoxification in honey bee (Berenbaum and Johnson 2015; Johnson et al. 2018). However, we did not detect any correlations between ecology and CYP clan sizes. Despite these limitations, we did uncover hints that AMP gene family size may be larger in non-eusocial lineages. If eusocial taxa tend to inhabit more sterile environments (nests and hives) than non-eusocial taxa, this finding is consistent with associations between habitat and AMP loss reported in dipterans (Hanson et al. 2019). Given that AMPs were also implicated in our within-genome analysis, immune-related genes are especially promising candidates for future manual annotation projects.

Comparative analysis is a powerful approach for evaluating the repeatability and predictability of evolutionary outcomes. Although our comparison of candidate gene family sizes among ecologically diverse hymenopterans hints at intriguing relationships between ecology and gene family size, it also had several limitations that should be revisited in future work. First, because several taxa in our manual annotation dataset are missing from published hymenopteran phylogenies (Peters et al. 2017), we were unable to correct for phylogenetic non-independence and polarize gene gain/loss (e.g., as in (Hahn et al. 2005; Han et al. 2013) without losing unacceptable amounts of data. Without accounting for similarity in ecology and gene family size due to recent common ancestry, our Type I error rate is likely inflated and p-values should be interpreted with caution. Nevertheless, variation in patterns of association among ecological traits and gene families suggest that phylogeny and ecology are, to some extent, decoupled.

The gene annotation and ecological datasets also had limitations. For example, across studies that included manual annotations, we observed a lack of consistency in the methods and criteria for manually curated gene family datasets. The most problematic inconsistency was in the criteria for delineating intact, 491 partial, and pseudogenized gene annotations. "Intact" could mean an exon-by-exon check against closely 492 related orthologs, a minimum amino acid length, or merely the presence of an expected domain.

493 Meanwhile, in reference publications, the number of pseudogenized and partial annotations were not 494 always reported or were conflated. This is in addition to variation in the methods used to search for genes. 495 Inconsistency in annotation methods and criteria across studies may introduce taxon-specific biases 
496 unrelated to ecology. Regarding ecology, categorizations are somewhat subjective. For example, this

497 study and Rane et al. (2016) classified bees as generalists since they collect nectar and pollen from

498 multiple plant families (we defined specialization as the use of a single taxonomic family). But Johnson et

499 al. (2018) classified bees as specialists as their diet consists of only nectar and pollen.

$500 \quad$ Finally, our attempts to correlate the size of different gene families with ecology suffered from

501 sampling biases in which species had genome assemblies and which gene families were manually

502 annotated. Species skewed heavily towards Drosophila and apocritan Hymenoptera, and annotations

503 toward the OR and CYP families (Table S8). To evaluate ecological correlates of gene family expansions

504 and contractions, it is essential to expand both the taxonomic breadth and depth of annotation sampling.

505 Taxa that capture independent ecological transitions (e.g., between herbivory and other diets) would be

506 especially useful, as would replicated groups of closely related species that vary in ecological axes of

507 interest (e.g., specialization or social behavior). By systematically sampling different ranges of divergence

508 times, we can evaluate the extent to which the tempo of gene family size change varies across different

509 gene families. To do so, however, will require high quality, manually curated datasets produced using

510 consistent methods and standards for many different environmentally responsive gene families.

\section{Conclusions}

Gene families that mediate ecological interactions may predictably expand and contract in

515 detectable genomic footprints in recent niche colonists and across taxa with convergent niche shifts.

516 Consistent with these predictions, (1) our analysis of gene family evolution in a derived pine feeder

517 suggests that expansions of GRs, CYP3s, and AMPs may have accompanied pine adaptation, and (2) our

518 comparison among ecologically diverse hymenopterans links two of these families to variation in diet

519 (GR) and eusociality (AMP). In the order Hymenoptera, the OR gene family was associated with ecology

520 (eusociality), however, the size of all five candidate gene families was not linked to other ecological axes

521 of variation (specialization/generalization); they were in other comparisons of closely related species

522 (McBride 2007)). Together, these results suggest that the size changes of environmentally responsive

523 gene families vary in both temporal dynamics (shallow vs. deep divergence times) and in ecological

524 drivers. Teasing apart these relationships will require high quality annotation data across diverse gene

525 families, ecologies, and divergence times. For hymenopterans, increased effort in understudied

526 symphytan, parasitoid, and herbivorous taxa would be especially useful for disentangling different axes of

527 ecological variation contributing to gene family size change. 


\section{Materials and methods}

530 Biological material

To minimize the confounding effects heterozygosity has on genome assembly, we sequenced

532 haploid siblings. Like all Hymenoptera, sawflies have haplodiploid sex determination in which males

533 (haploid genomes) emerge from unfertilized eggs and females (diploid genomes) from fertilized eggs. A

534 virgin female will bear a clutch of all-male offspring with haploid recombinants of the maternal genome.

535 But the individual genomes are not identical, so an assembly derived from a single clutch is akin to a

536 diploid assembly made from a single individual.

All insects were reared in custom, climate-controlled environmental chambers (18:6 light cycle, $22^{\circ} \mathrm{C}, 70 \% \mathrm{RH}$ ) on jack pine (Pinus banksiana) foliage. Our laboratory line of $N$. lecontei was established from multiple larval colonies collected from a mugo pine (P. mugo) in Lexington, Kentucky, USA

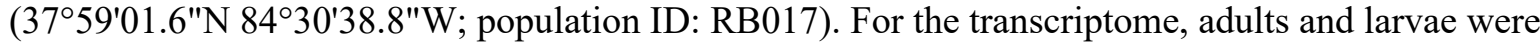
collected from the first lab-reared generation; both were stored at $-80^{\circ} \mathrm{C}$. For the genome assembly, the

542 founding population was propagated in the lab for two generations, followed by brother-sister matings for 543 an additional two generations. At this point, a single, virgin, adult female (I2G2-V, $4^{\text {th }}$ generation in the 544 lab) was allowed to lay unfertilized eggs onto jack pine seedlings. The offspring (haploid male brothers 545 from an inbred mother) were reared until the eonymph (prepupal) life stage, at which point they were isolated without food for 24 hours prior to preservation in absolute ethanol at $-20^{\circ} \mathrm{C}$. Although eonymphs are non-feeding, they were starved to ensure the gut contents were completely voided.

548 Sample preparation and sequencing

Genomic DNA

Whole eonymph bodies were individually frozen inside microcentrifuge tubes with liquid nitrogen and ground with pestles made from 1-mL micropipette tips; the resulting powder was incubated in CTAB buffer supplemented with proteinase K and RNase A. After PCI extraction and ethanol precipitation, the precipitate was dried overnight before being resuspended in TE buffer. DNA integrity was assessed with $0.7 \%$ agarose gel, purity was measured with the $260 / 280$ ratio, and concentration was measured with a Quant-iT dsDNA High-Sensitivity fluorescence assay (Thermo Fisher Scientific).

The HudsonAlpha Genomic Services Lab (Huntsville, AL, USA) prepared and sequenced the DNA libraries. Two small-insert, barcoded libraries with average fragment sizes of $337 \mathrm{bp}$ and $864 \mathrm{bp}$ were made from a single individual. A 4.6-kbp mate-pair, barcoded library was made from 25 pooled individuals. All individuals were brothers from the same I2G2-V mother. The libraries were sequenced on Illumina HiSeq 2000 with paired-end, 100 bp (PE100) reads: the small-insert libraries each had $1 / 4$ of a

561 flow cell lane and the mate-pair library had an entire lane.

$562 m R N A$ 
The RNeasy Mini extraction kit (Qiagen) was used to collect total RNA from adult female body, adult female head, adult male body, adult male head, eonymph body, feeding larval body, and feeding larval head. RNA from eonymph head was extracted but not sequenced due to insufficient yield. Each tissue was represented with one replicate that had equal RNA contributions from eight individuals, except for eonymph body which was comprised of three individuals. RNA integrity and concentration were measured with a 2100 Bioanalyzer (Agilent).

The HudsonAlpha Genomic Services Lab (Huntsville, AL, USA) handled library preparation and sequencing. Non-stranded, barcoded libraries were made for each of the seven tissue samples; on average, mRNA was sheared to $200 \mathrm{bp}$. The libraries were combined and sequenced on an entire flow cell of Illumina MiSeq with PE250 reads in addition to one lane of Illumina HiSeq 2000 with PE50 reads.

\section{Read processing and assembly}

De novo genome assembly

Sequencing reads were chastity-filtered and adaptor-trimmed with fastq-mcf (ea-utils v1.04.803) (Aronesty 2011), and quality-filtered with fastq_quality_filter (FASTX Toolkit v0.0.13.2) (Gordon and Hannon 2019). The 337-bp small-insert reads and the 4.6-kbp mate-pair reads were quality-filtered to retain reads where at least $80 \%$ of the bases had a quality score of 20 or higher (parameters: $-q 20-p$ 80). Due to sequencing quality, the 864-bp small-insert reads were filtered to retain reads where at least $70 \%$ of the bases had a quality score of 20 or higher (R1) or $60 \%(R 2)$ (parameters: $-q 20-p$ $60 / 70$ ). In situations where only one end of the paired-end reads passed filtering, the passed reads were kept and treated as single-end data. Kmer counting was used to measure read depth before and after filtering (Jellyfish v1.1.11) (Marçais and Kingsford 2011). Finally, reads were screened for sequencing contamination by mapping the reads (BWA v0.7.12-r1039) (Li and Durbin 2009) to reference genomes for Escherichia coli (K12 substr. DH10B uid58979), human (v37), loblolly pine (Pinus taeda, v0.8), and Wolbachia (endosymbiont of Dmel uid57851).

The genome was assembled with ALLPATHS-LG (v47417) (Gnerre et al. 2011) using default settings, including a minimum scaffold size of $1000 \mathrm{bp}$. The error-correction module was run on the reads prior to assembly. After assembly, GapFiller (v1.11) (Boetzer and Pirovano 2012) was used to help close

590 intra-scaffold gaps. Spurious scaffolds were identified with SOAP.coverage (v2.7.7) (Li et al. 2009):

591 reads were mapped to the assembly scaffolds and scaffolds with a read depth $<15$ and nucleotide

592 percentage $<40$ were removed. The completeness of the final assembly was measured with CEGMA

593 (v2.5) (Parra et al. 2007) and BUSCO (v1.22) (Simão et al. 2015) benchmarks. BUSCO was run with the 594 arthropoda-25oct16 database (parameters: --long). 
For both the PE250 MiSeq and the PE50 HiSeq reads, fastq-mcf (ea-utils v.1.04.803) (Aronesty 2011) was used for chastity filtering and Trimmomatic (v0.32) (Bolger et al. 2014) was used to adaptor clip, trim, and quality-filter. The PE250 MiSeq reads were processed with the Trimmomatic parameters ILLUMINACLIP: 2:15:5, HEADCROP: 10, CROP: 60, MINLEN: 60, AVGQUAL: 25 whereas the PE50 HiSeq reads were processed with ILLUMINACLIP : 2:15:5, HEADCROP : 15, MINLEN : 35, AVGQUAL : 25. Because the mRNA libraries had an average insert size of $200 \mathrm{bp}$, the MiSeq reads required extensive adaptor trimming. Reads were screened for contamination as described in De novo genome assembly.

For each tissue, transcriptomes were assembled with Trinity (r2013_08_14) (Grabherr et al. 2011; Haas et al. 2013) using default settings and the--jaccard_clip option. Spurious sequences were identified by mapping the sequencing reads to the assembled transcripts with RSEM (v1.2.18) (Li and Dewey 2011); transcripts with either FPKM or TPM values $<1$ were removed. After filtering, the transcriptomes were combined, and duplicate sequences were removed.

\section{Genome size estimation}

$610 \quad$ Flow cytometry was described in (Harper et al. 2016). For this analysis, we used adult males and females from a lab line of $N$. lecontei established from a colony collected in Auburn,

612 GA (3359'22.4" N, 8347'44.6"W; population ID: RB027). Briefly, cell nuclei were collected

613 from the heads of 7 individuals (4 female, 3 male) and stained with propidium iodide. Mean

614 fluorescence for each sample was measured with a BD FACSCalibur flow cytometer (BD

615 Biosciences) and compared to two external standards: Drosophila melanogaster (adult female

616 heads, $1 \mathrm{C}=175 \mathrm{Mbp}$ ) and Gallus gallus domesticus (CEN singlets from BioSure, Grass Valley,

$617 \mathrm{CA}, 1 \mathrm{C}=1222.5 \mathrm{Mbp}$ ). To correct for ploidy differences between haploid males and diploid

618 standards, we multiplied the $N$. lecontei male estimates by 2 . To obtain a single size estimate for 619 each N. lecontei sample, we averaged values obtained for the two standards.

\section{Repeat annotation}

The N. lecontei genome assembly was masked with a custom repeat library. A lineage-

623 2015) and combined with the hymenopteran repetitive element database (Nov. 2013) from

624 Repbase (Jurka et al. 2005). The custom library was used by RepeatMasker (v4.0.3) (Smit et al.

625 2013-2015) (parameters: -cutoff 250 -s -pa 15 -gc 40 -a -poly) to identify and

626 mask repetitive elements in the genome, including low-complexity DNA and simple repeats. 
Transposable element (TE) family consensus sequences were identified by rerunning

628 RepeatModeler (Smit and Hubley 2008-2015) on the genome assembly using the "ncbi" search engine.

629 The resulting sequences were provided to RepeatMasker (Smit et al. 2013-2015) as a custom library to

630 locate associated TE copies in the genome (parameters: -gc 40 -cutoff 250 -gff -gccalc -

631 norna -nolow -no_is -poly). TE families with at least 10 fragments longer than $100 \mathrm{bp}$ were

632 extracted for further analysis.

633 The sequencing reads were mapped to a concatenation of the masked genome and the consensus

634 TE sequences (BWA MEM (parameters: -M) (Li and Durbin 2009)). Families that had at least 1x the

635 median coverage to the reference genome for at least $80 \%$ of their sequence (to support at least one full

636 insertion found by RepeatModeler) and at least $2 \mathrm{x}$ the maximum coverage of the reference genome (to

637 support multiple insertions of the family) were extracted with genomeCoverageBed (BEDtools (Quinlan

638 and Hall 2010)). We attempted to identify the consensus sequences with BLASTN and BLASTX

639 (Altschul et al. 1990) searches against a database of repeat elements, but the only hits were to the lineage-

640 specific elements identified by RepeatModeler. Sequences were also filtered for BLAST hits to rRNA or

641 mitochondrial sequences.

642 We also used dnaPipeTE (Goubert et al. 2015) to identify what proportion of our short reads was

643 composed of repetitive content, we used a random subset of reads corresponding to 1-fold coverage of the

644 genome $(331 \mathrm{Mb})$ and took the total for three separate random samplings of reads (parameters: genome

645 size $=331000000$ genome coverage $=1$ samples number $=3$ ). We then compared this annotation to the

646 RepeatModeler annotation.

647 Gene and functional annotation

648 Automated gene annotation

649 RNA-Seq data for $N$. lecontei was used to generate training models for gene prediction along with

650 utilization of peptide sequences from other species. PASA (r20130425beta) was used to build a

651 comprehensive transcriptome set from Trinity assembled transcripts along with RNA-Seq read mapping

652 predictions generated from the Tuxedo pipeline. To improve annotation quality, in addition to this $N$.

653 lecontei transcriptome, annotated proteins from Atta cephalotes (OGSv1.2), Acromyrmex echinatior

654 (OGSv3.8), Apis melifera (OGSv3.2), Athalia rosae (OGSv1.0), and Nasonia vitripennis (OGSv1.0) were

655 provided to Maker (2.09) (Cantarel et al. 2008) as evidence for structural gene prediction. Prior to

656 annotation, the genome was masked using a custom repeat database built using RepeatModeler (v1.0.8)

657 and the annotation was run using the ab initio gene predictors Augusts, Genemark-ES and snap in

658 addition to the evidence provided. The functions of the predicted protein-coding genes were putatively

659 established with BLASTP alignments (Altschul et al. 1990) to the Swiss-Prot database (accessed 20 Apr

660 12) (Apweiler et al. 2004). In cases of multiple matches, the top-ranked alignment was assigned to the 
661 gene annotation. Protein motifs and functional domains within the annotations were also identified with

662 an InterProScan (v5.3.46.0) (Jones et al. 2014) search against the InterPro database with gene ontology

663 and IPR lookup (Finn et al. 2016). For the official gene set (OGS), the Maker annotations were filtered by

664 hits to the reference databases and/or a minimum eAED score of 0.1 . A second set of gene annotations

665 was generated with the NCBI GNOMON pipeline (annotation release 100 on Nlec1.0 assembly,

666 GCF_001263575.1) (Souvorov et al. 2010).

667 As the genome was annotated prior to submission to NCBI, we encountered a problem when the

668 NCBI contamination software flagged vector/adaptor sequences for removal; this would disrupt the

669 coordinates provided by Maker. We used a modified version of GAG (Hall et al. 2014) that could accept

670 the flagged coordinates from NCBI to edit the assembly and update annotation coordinates accordingly.

671 Chemoreceptor genes

672 The olfactory (OR) and gustatory (GR) receptor genes were manually curated following

673 Robertson et al. (2003, 2006). Amino acid sequences of manually curated chemoreceptor genes from Apis

674 mellifera (Robertson and Wanner 2006; Smith et al. 2011), Bombus terrestris (Sadd et al. 2015) and

675 Cephus cinctus (Robertson et al. 2018), Drosophila melanogaster (Flybase release FB2017_04), and

676 Nasonia vitripennis (Robertson et al. 2010) were used as queries in TBLASTN (v2.2.19) (Altschul et al.

677 1990) searches against the $N$. lecontei draft genome (parameters: -e 100000 -F F). Gene models

678 were manually built in TextWrangler (v5.5) (Bare Bones Software), using protein alignment to identify

679 exons and refine the gene structures; alignments were visualized with Clustal X (v2.1) (Larkin et al.

680 2007). The Neural Network Splice Predictor program from the Berkeley Drosophila Genome Project was

681 used to help identify intron splice sites (http://www.fruitfly.org/seq_tools/splice.html). New gene models

682 were added to TBLASTN searches and this process continued iteratively until new chemoreceptors were

683 no longer found. The gene models were checked against RNAseq reads from tissue-specific

684 transcriptomes (adult antennae, mouthparts, heads, legs, genitalia, and larval heads (Herrig et al. 2019))

685 and against orthologs in the N. pinetum draft genome assembly (NCBI accession GCA_004916985.1).

686 Odorant binding proteins

687 Custom scripts were used to identify Maker gene annotations (see Automated gene annotation)

688 that contained the classic/6C, Plus-C, Minus-C, or atypical odorant binding protein (OBP) motif (Xu et al.

689 2009). These as well as OBPs from Apis mellifera and Nasonia vitripennis were used as queries for

690 TBLASTN searches against the N. lecontei genome; searches did not yield any new OBPs. All genomic

691 regions identified as potential OBPs were manually curated as described for chemoreceptor genes. After

692 manual annotation, duplicate annotations or genes that lacked OBP motifs were removed.

693 Cytochrome P450 genes

694 A broad set of 52 insect CYP genes (covering the diversity of insect CYP families) were 
searched against the N. lecontei genome assembly (E-value cutoff 1e3). Scaffolds with hits were then searched against 8782 known insect CYPs. The top 10 hits were returned (later increased to 15 to recover more sequences) and filtered for duplicates. An alternative search of the NCBI GNOMON predictions

698 ("Neodiprion lecontei[orgn] AND P450 NOT reductase") was also performed and new sequences were

699 added to the dataset. This approach found all the loci identified by the initial search, indicating that the

700 GNOMON annotation tool was able to comprehensively search for CYP sequences. Finally, the candidate

701 N. lecontei CYP sequences were manually curated based on comparison to the best BLAST hits.

702 Immune-related genes

703 Because of the relative completeness of its immune annotation, Drosophila melanogaster

704 immunity genes were used to guide annotation. Reference immune genes from D. melanogaster tagged

705 with the gene ontology term "GO:0002376 - Immune system process" were compiled from Flybase

706 (release 6.13). Orthology with N. lecontei proteins was assigned initially with reciprocal BLASTP

707 (Altschul et al. 1990) searches (E-value cutoff 1e-10). Reference D. melanogaster genes without obvious

708 one-to-one orthologs in N. lecontei were examined individually to determine whether closely related

709 paralogs in one or both species interfered with the inference of orthology. If not, they were searched

710 against the $N$. lecontei genome assembly using TBLASTN (Altschul et al. 1990) in an attempt to identify

711 unannotated orthologs.

712 Since antimicrobial peptides (AMP) are unlikely to be conserved between D. melanogaster and

713 N. lecontei, AMPs from three representative hymenopterans Apis mellifera (Danihlík et al. 2015),

714 Nasonia vitripennis (Tian et al. 2010), and Camponotus floridanus (Ratzka et al. 2012; Zhang and Zhu

715 2012; Gupta et al. 2015) were used for BLAST queries. Furthermore, since AMP copy number is fast

716 evolving, we attempted to find all the $N$. lecontei orthologs of each hymenopteran AMP instead of

717 focusing on one-to-one orthology. Once again, BLASTP searches were performed against the annotated

718 proteins and TBLASTN searches were performed against the assembled genome; the TBLASTN search

719 did not reveal additional AMPs. Putative N. lecontei orthologs were reciprocally blasted against the

720 appropriate hymenopteran proteome to assure that the best hits were indeed AMPs.

721 Amino acid and cDNA sequences for all manual annotated genes are available in File S1.

\section{Glomeruli counts}

723 Antennal lobe histology

724 Whole heads of adult $N$. lecontei of both sexes were fixed in $2 \%$ paraformaldehyde, $2 \%$

725 glutaraldehyde in PBS for 5 days. Heads were rinsed for 40 minutes three times and the brains dissected

726 out in cold PBS. Following blocking with goat serum, brains were permeabilized with 1\% Triton X-100

727 in PBS (Electron Microscopy Supply, Fort Washington, PA; PBS-TX ), rinsed with 0.1\% PBS-TX, and

728 incubated on a shaker at $25^{\circ} \mathrm{C}$ for three nights in primary antibody ( $1: 500$ in $2 \%$ goat serum in $0.2 \%$ PBS- 
TX). Monoclonal Drosophila synapsin I antibody (SYNORF1, AB_2315426) from the Developmental Studies Hybridoma Bank (catalog 3C11) was used to label synapsin. Subsequently, brains were washed in $0.1 \%$ PBS-TX and incubated for two nights in Alexa Fluor 568 (ThermoFisher) goat anti-mouse

732 secondary antibody (1:100 in PBS) in the dark at room temperature on a shaker. After secondary

733 incubation, brains were rinsed with distilled water, dehydrated in increasing concentrations of ethanol,

734 and mounted in custom-made aluminum well slides. Brains were cleared by removing ethanol and

735 replacing it with methyl salicylate. Brains were imaged on an inverted Zeiss 880 Laser Scanning

736 Confocal Microscope with a 20X plan-Apochromat 20x 0.8 aperture objective and optically sectioned in 737 the horizontal plane at 3-micron intervals.

\section{Glomeruli segmentation}

Whole-brain images of one female and one male were manually segmented using the TrakEM2 software package in ImageJ (Cardona et al. 2012; Schindelin et al. 2012). Individual glomeruli were traced in both brain hemispheres. Glomeruli near the center of the antennal lobe can be difficult to

742 distinguish, meaning counts are biased toward fewer glomeruli and the largest number of glomeruli confidently detected represents a minimum of the number of expected glomeruli. Male Neodiprion have a collection of smaller synaptic clusters in their antennal lobe (Dacks and Nighorn 2011), but the functional significance of this anatomy is not known. There are more than 50 of these smaller synaptic clusters and we suspect they do not represent the traditional one-to-one OR-to-glomerulus organization. Therefore, these structures were not included in counts. Male glomeruli number may be lower if particular OSNs contribute to these clusters instead of forming traditional glomeruli.

\section{Within-genome signatures of adaptive expansions and contractions}

$750 \quad$ Clustering and pseudogene analyses

To evaluate the extent to which members of our five focal gene families were located in tandem

752 arrays, we placed our annotated genes on a linkage-map anchored version of the $N$. lecontei genome

753 assembly described in Linnen et al. 2018. We considered genes to be clustered if they were located within

754 a genomic region of 20(n-1) kilobases, where $n$ is the number of genes in the cluster under

755 consideration. This criterion was chosen based on average gene densities in Nasonia (Niehuis et al. 2010)

756 and clustering criteria described Drosophila (Vieira et al. 2007). For scaffolds that could not be placed on

757 linkage groups, we evaluated clustering only if genes were more than $20 \mathrm{~kb}$ from either scaffold end.

758 To evaluate whether the five focal gene families differed in (1) the proportion of genes found in

759 clusters of two or more or (2) the proportion of pseudogenized genes, we performed Fisher's exact tests in

760 R v3.5.0 (“fisher.test" function) (R-Core-Team 2018). For significant Fisher's exact tests, we performed

761 additional posthoc tests using the "fisher.multcomp" function (from R package RVAideMemoire v. 0.9-

762 72) with FDR correction (Benjamini-Hochberg method) for multiple comparisons. 
Identification of Neodiprion-specific clades and tests of positive selection

First, we identified clades unique to $N$. lecontei. For each gene family, a multi-species, amino acid phylogeny was constructed with manually curated annotations from $N$. lecontei, select Hymenoptera, and D. melanogaster. Sequences were size filtered (350 $\geq$ for GR, OR, CYP; $100 \geq$ for histnavicin and OBP), but pseudogenes and partial annotations that met the length requirement were retained. MAFFT alignments (v7.305b) (Katoh et al. 2002) (parameters: --maxiterate 1000 -localpair) were visually inspected to remove sequences with large alignment gaps, and sites with more than $20 \%$ gaps were removed with trimAl (v1.4.rev15 build[2013-12-17]) (Capella-Gutiérrez et al. 2009) (parameters: -gapthreshold 0.8). Maximum likelihood phylogenies were made in RAxML (v8.2.4) (Stamatakis 2014) (parameters: -f a $-x 12345$-p 12345 -\# autoMRE) using protein substitution models chosen from ProtTest3 (v3.4.2) (Abascal et al. 2010; Darriba et al. 2011).

Neodiprion-specific clades were defined as those with at least five N. lecontei genes (not including partial and pseudogenes) and a bootstrap score $\geq 70$ (Engsontia et al. 2015). Second, the clades were confirmed with cDNA phylogenies for each $N$. lecontei gene family. Amino acid sequences were aligned as above, however, after alignment TranslatorX (Abascal et al. 2010) was used to map cDNA sequences to the amino acid alignment. After trimming, the cDNA alignments were passed to RAxML to construct maximum likelihood gene family trees with the nucleotide substitution model $-m$ GTRGAMMA.

Site tests were conducted with codeml (part of the PAML package (PAML v4.9e) (Yang 2007)) family cDNA phylogeny was pruned to remove all branches except for that clade. Codeml models M7, M8, and M8a were fitted to the cDNA sequence and phylogeny data. Likelihood-ratio tests were performed for the nested models M7-M8 (null model M7 that equally distributes amino acid sites across 10 classes of $\omega$ parameter values $(\mathrm{p}, \mathrm{q})$ against alternative model M8 that has an $11^{\text {th }}$ class for positively selected sites) and M8-M8a (null model M8a that has 11 classes and does not allow positive selection

787 against alternative model M8). Bonferroni correction was applied to the likelihood-ratio test probability

788 values; each value was multiplied by two since two tests that used M8 as the alternative model were 789 performed on each clade. For clades with significant likelihood-ratio tests, sites under selection were 790 identified by looking at the Bayes Empirical Bayes analysis within the alternative models.

For branch tests, the cDNA phylogenies for each $N$. lecontei gene family were used to compare

792 the lineage-specific clade to the rest of the gene family. To determine if the foreground branch $\mathrm{dN} / \mathrm{dS}$

793 (i.e., the branch with the species-specific expansion) was significantly different from the background (i.e., 794 the rest of the gene family), in codeml we ran a two-ratio model (Model=2, fix_omega=0) and a one-ratio model (Model=0, fix_omega $=0$ ) for that clade and performed a likelihood-ratio test comparing the two 
models. To determine if the foreground branch is under positive selection $(\mathrm{dN} / \mathrm{dS}>1)$, we performed a

797 likelihood-ratio test comparing the two-ratio model to a neutral model (fix_omega=1).

798 Ecological correlates of gene family size among insects

All the insect genome assembly projects we could find (published and unpublished) were

800 searched for manually curated OR, GR, OBP, CYP, and AMP gene annotations. If fasta sequence files

801 were available, the number of intact, partial, and pseudogenized genes was determined by gene names

802 (e.g., labels with "pse" or "partial") and compared to values reported in the publication. Otherwise, we

803 relied on reported values. If gene family size was reported but not broken down into intact, partial, and

804 pseudogenized, and sequence files were unavailable, we assumed that the reported number referred to

805 intact genes. Splice variants were not included in the gene count. It is important to note that different

806 authors likely used different criteria for these categories.

Only putatively functional (intact) gene were used in gene family size comparisons. Species were classified according to taxonomic order, diet type, dietary specialization, and sociality. An order needed at least two species to be included. Specialization was defined as the use of a single taxonomic family and only referred to the realized diet niche, ignoring reports of feeding under laboratory conditions. If a

811 species had a preferred host or both specialist and generalist life stages, it was classified as specialist.

812 Comparisons were made in R (v3.5.0) where species were grouped by the different classifications.

813 Because both gene family size and ecology are likely to correlate with phylogeny, the ideal

814 approach to identifying ecological correlates of gene family evolution is to use statistical methods that

815 account for phylogenetic relationships (Hahn et al. 2005; De Bie et al. 2006; Han et al. 2013).

816 Unfortunately, a lack of overlap between species with manual annotations for our focal gene families and

817 species included in published hymenopteran genomes precluded us from such an analysis without a

818 substantial loss of sample size. Therefore, as a first step to evaluating ecological correlates of gene family

819 size, we used non-parametric tests to determine whether gene family size differed among taxa. For

820 sociality and specialization, we used two-tailed Wilcoxon rank-sum tests ("wilcox.exact" function in the

821 exactRankTests v0.8-30 package). For taxonomic order and diet, both of which have more than two

822 categories, we used Kruskal-Wallis tests ("kruskal.test" function) followed by Dunn's post-hoc tests of

823 multiple comparisons (“dunnTest" function in the FSA v0.8.23 package).

\section{Acknowledgements}

We thank Linnen lab members for insect collection, insect reading, and reading earlier

827 manuscripts; Jeramiah Smith, Erin Scully, and Romain Studer for advice. We are especially grateful to

828 Hugh Robertson for his guidance on manual chemoreceptor gene annotation. This work was supported by

829 the University of Kentucky Center for Computational Sciences and the Lipscomb High Performance 
830 Computing Cluster, the United States Department of Agriculture National Institute of Food and

831 Agriculture (2016-67014-2475; CRL), the Kentucky Science and Engineering Foundation (KSEF-3492-

832 RDE-019; CRL), and the University of Kentucky (Lyman T. Johnson Fellowship; KV).

\section{Data availability}

The genome assembly, official gene set (OGS), and transcriptome described in this paper (v1 versions) can be found at https://i5k.nal.usda.gov/neodiprion-lecontei

On GenBank (NCBI), the genome assembly is labeled whole genome shotgun sequencing project accession PRJNA28045 and the genomic sequencing reads are RefSeq accession PRJNA312506. The transcriptome is transcriptome shotgun assembly accession GEDM00000000; this is a combined transcriptome of all seven tissue types. The mRNA sequencing reads for each tissue type was submitted separately under BioSample and short read archive accessions SAMN04302192 (adult female head),

842 SAMN04302193 (adult female body), SAMN04302194 (adult male head), SAMN04302195 (adult male

843 body), SAMN04302196 (feeding larval head), SAMN04302197 (feeding larval body), and

844 SAMN04302198 (eonymph body). The predicted gene annotations on NCBI are from Gnomon, the NCBI

845 annotation pipeline, and were not described in this paper. Finally, the clustering analysis was based on a

846 linkage-map anchored version of the genome assembly described in Linnen et al. 2018. This anchored

847 assembly is denoted as v1.1 in NCBI and the N. lecontei i5k Workspace@NAL (USDA).

\section{References}

850 Abascal F, Zardoya R, Telford MJ. 2010. TranslatorX: multiple alignment of nucleotide sequences guided by amino acid translations. Nucleic Acids Res 38(suppl 2):W7-W13. organization and rate of evolution of wheat genomes are correlated with recombination rates along chromosome arms. Genome Res 13(5):753-763.

Altschul SF, Gish W, Miller W, Myers EW, Lipman DJ. 1990. Basic local alignment search tool. J Mol Biol 215(3):403-410.

Apweiler R, Bairoch A, Wu CH, Barker WC, Boeckmann B, Ferro S, Gasteiger E, et al. 2004. UniProt: the universal protein knowledgebase. Nucleic Acids Res 32(suppl 1):D115-D119.

862 Aronesty E. 2011. ea-utils : "Command-line tools for processing biological sequencing data". 
Berenbaum MR. 2002. Postgenomic chemical ecology: from genetic code to ecological interactions. J Chem Ecol 28(5):873-896.

Berenbaum MR, Johnson RM. 2015. Xenobiotic detoxification pathways in honey bees. Curr Opin Insect Sci 10:51-58.

Boetzer M, Pirovano W. 2012. Toward almost closed genomes with GapFiller. Genome Biol 13(6):R56.

Boevé J-L, Blank SM, Meijer G, Nyman T. 2013. Invertebrate and avian predators as drivers of chemical defensive strategies in tenthredinid sawflies. BMC Evol Biol 13(1):198.

Bolger AM, Lohse M, Usadel B. 2014. Trimmomatic: a flexible trimmer for Illumina sequence data. Bioinformatics 30(15):2114-2120.

Brand P, Ramírez SR. 2017. The evolutionary dynamics of the odorant receptor gene family in corbiculate bees. Genome Biol Evol 9(8):2023-2036.

Briscoe AD, Macias-Munoz A, Kozak KM, Walters JR, Yuan F, Jamie GA, Martin SH, et al. 2013. Female behaviour drives expression and evolution of gustatory receptors in butterflies. PLoS Genet 9(7):e1003620.

Calla B, Noble K, Johnson RM, Walden KKO, Schuler MA, Robertson HM, Berenbaum MR. 2017. Cytochrome P450 diversification and hostplant utilization patterns in specialist and generalist moths: Birth, death and adaptation. Mol Ecol 26(21):6021-6035.

Cantarel BL, Korf I, Robb SMC, Parra G, Ross E, Moore B, Holt C, Alvarado AS, Yandell M. 2008. MAKER: an easy-to-use annotation pipeline designed for emerging model organism genomes. Genome Res 18(1):188-196.

Cao D, Liu Y, Walker WB, Li J, Wang G. 2014. Molecular characterization of the Aphis gossypii olfactory receptor gene families. PLoS One 9(6):e101187.

Capella-Gutiérrez S, Silla-Martínez JM, Gabaldón T. 2009. trimAl: a tool for automated alignment trimming in large-scale phylogenetic analyses. Bioinformatics 25(15):1972-1973.

Cardona A, Saalfeld S, Schindelin J, Arganda-Carreras I, Preibisch S, Longair M, Tomancak P, Hartenstein V, Douglas RJ. 2012. TrakEM2 software for neural circuit reconstruction. PLoS One 7(6):e38011.

Castella G, Chapuisat M, Moret Y, Christe P. 2008. The presence of conifer resin decreases the use of the immune system in wood ants. Ecological Entomology 33(3):408-412.

895 Consortium iK. 2013. The i5K Initiative: advancing arthropod genomics for knowledge, human health, nonrandom subset of olfactory genes is associated with host preference in the fruit fly Drosophila orena. Evolution Letters 1(2):73-85.

agriculture, and the environment. J Hered, 104(5):595-600. 
Couto A, Alenius M, Dickson BJ. 2005. Molecular, anatomical, and functional organization of the Drosophila olfactory system. Curr Biol 15(17):1535-1547.

Cowan MM. 1999. Plant products as antimicrobial agents. Clin Microbiol Rev 12(4):564-582.

Dacks AM, Nighorn AJ. 2011. The organization of the antennal lobe correlates not only with phylogenetic relationship, but also life history: a basal hymenopteran as exemplar. Chem Senses 36(2):209-220.

Dahanukar A, Foster K, Carlson JR. 2001. A Gr receptor is required for response to the sugar trehalose in taste neurons of Drosophila. Nat Neurosci 4(12):1182-1186.

Danihlík J, Aronstein K, Petřivalský M. 2015. Antimicrobial peptides: a key component of honey bee innate immunity: Physiology, biochemistry, and chemical ecology. Journal of Apicultural Research 54(2):123-136.

Darriba D, Taboada GL, Doallo R, Posada D. 2011. ProtTest 3: fast selection of best-fit models of protein evolution. Bioinformatics 27(8):1164-1165.

Dayhoff MO. 1976. The origin and evolution of protein superfamilies Federation Proceedings 35(10):2132-2138.

De Bie T, Cristianini N, Demuth JP, Hahn MW. 2006. CAFE: a computational tool for the study of gene family evolution. Bioinformatics 22(10):1269-1271.

915 Demuth JP, Hahn MW. 2009. The life and death of gene families. Bioessays 31(1):29-39.

916 Després L, David J-P, Gallet C. 2007. The evolutionary ecology of insect resistance to plant chemicals.

917 Trends Ecol Evol 22(6):298-307.

918 Dobler S, Dalla S, Wagschal V, Agrawal AA. 2012. Community-wide convergent evolution in insect

919 adaptation to toxic cardenolides by substitutions in the Na, K-ATPase. Proc Natl Acad Sci USA

920 109(32):13040-13045.

Duffy JE, Macdonald KS. 2010. Kin structure, ecology and the evolution of social organization in shrimp:

922 a comparative analysis. Proc Royal Soc B 277(1681):575-584.

923 Engsontia P, Sangket U, Robertson HM, Satasook C. 2015. Diversification of the ant odorant receptor

924 gene family and positive selection on candidate cuticular hydrocarbon receptors. BMC Res Notes

$925 \quad 8(1): 380$.

926 Evans JD, Aronstein K, Chen YP, Hetru C, Imler JL, Jiang H, Kanost M, Thompson GJ, Zou Z, Hultmark

927 D. 2006. Immune pathways and defence mechanisms in honey bees Apis mellifera. Insect Mol Biol

928 15(5):645-656.

929 Faulkes CG, Bennett NC, Bruford MW, O’Brien HP, Aguilar GH, Jarvis JU. 1997. Ecological constraints

930 drive social evolution in the African mole-rats. Proc Royal Soc B 264(1388):1619-1627. 
Feyereisen R. 2011. Arthropod CYPomes illustrate the tempo and mode in P450 evolution. Biochimica et Biophysica Acta (BBA)-Proteins and Proteomics 1814(1):19-28.

Feyereisen R. 2012. Insect CYP genes and P450 enzymes. In. Insect Mol Biol and Biochemistry: Academic Press. p. 236-316.

Finn RD, Coggill P, Eberhardt RY, Eddy SR, Mistry J, Mitchell AL, Potter SC, et al. 2016. The Pfam protein families database: towards a more sustainable future. Nucleic Acids Res 44(D1):D279-D285.

Fischman BJ, Woodard SH, Robinson GE. 2011. Molecular evolutionary analyses of insect societies. Proc Natl Acad Sci USA 108(suppl 2):10847-10854.

Fishilevich E, Vosshall LB. 2005. Genetic and functional subdivision of the Drosophila antennal lobe. Curr Biol 15(17):1548-1553.

Forister ML, Dyer LA, Singer MS, Stireman III JO, Lill JT. 2012. Revisiting the evolution of ecological specialization, with emphasis on insect-plant interactions. Ecology 93(5):981-991.

Gao Q, Yuan B, Chess A. 2000. Convergent projections of Drosophila olfactory neurons to specific glomeruli in the antennal lobe. Nat Neurosci 3(8):780-785.

Gardiner A, Barker D, Butlin RK, Jordan WC, Ritchie MG. 2008. Drosophila chemoreceptor gene evolution: selection, specialization and genome size. Mol Ecol 17(7):1648-1657.

Gaut BS, Wright SI, Rizzon C, Dvorak J, Anderson LK. 2007. Recombination: an underappreciated factor in the evolution of plant genomes. Nat Rev Genet 8(1):77-84.

$950 \quad$ Chem Biol 3(7):408-414.

Gnerre S, MacCallum I, Przybylski D, Ribeiro FJ, Burton JN, Walker BJ, Sharpe T, et al. 2011. Highquality draft assemblies of mammalian genomes from massively parallel sequence data. Proc Natl Acad Sci USA 108(4):1513-1518.

Goldman-Huertas B, Mitchell RF, Lapoint RT, Faucher CP, Hildebrand JG, Whiteman NK. 2015. Evolution of herbivory in Drosophilidae linked to loss of behaviors, antennal responses, odorant receptors, and ancestral diet. Proc Natl Acad Sci USA 112(10):3026-3031.

Good RT, Gramzow L, Battlay P, Sztal T, Batterham P, Robin C. 2014. The molecular evolution of cytochrome P450 genes within and between Drosophila species. Genome Biol Evol 6(5):1118-1134. Gordon A, Hannon GJ. 2019. "Fastx-toolkit" FASTQ/A short-reads preprocessing tools (unpublished). assembly and annotation of the Asian tiger mosquito (Aedes albopictus) repeatome with dnaPipeTE

963 Genome Biol Evol 7(4):1192-1205. 
Grabherr MG, Haas BJ, Yassour M, Levin JZ, Thompson DA, Amit I, Adiconis X, et al. 2011. Fulllength transcriptome assembly from RNA-Seq data without a reference genome. Nat Biotechnol 29(7):644-652.

Grayer RJ, Harborne JB. 1994. A survey of antifungal compounds from higher plants, 1982-1993. Phytochemistry 37(1):19-42.

Gross JB, Borowsky R, Tabin CJ. 2009. A novel role for Mc1r in the parallel evolution of depigmentation in independent populations of the cavefish Astyanax mexicanus. PLoS Genet 5(1):e1000326.

Gupta SK, Kupper M, Ratzka C, Feldhaar H, Vilcinskas A, Gross R, Dandekar T, Förster F. 2015. Scrutinizing the immune defence inventory of Camponotus floridanus applying total transcriptome sequencing. BMC Genom 16(1):1-21.

Ha E-M, Oh C-T, Bae YS, Lee W-J. 2005. A direct role for dual oxidase in Drosophila gut immunity. Science 310(5749):847-850.

Haas BJ, Papanicolaou A, Yassour M, Grabherr MG, Blood PD, Bowden J, Couger MB, et al. 2013. De novo transcript sequence reconstruction from RNA-seq using the Trinity platform for reference generation and analysis. Nat Protoc 8(8):1494-1512.

Hahn MW, de Bie T, Stajich JE, Nguyen C, Cristianini N. 2005. Estimating the tempo and mode of gene family evolution from comparative genomic data. Genome Res 15(8):1153-1160.

Hahn MW, Han MV, Han S-G. 2007. Gene family evolution across 12 Drosophila genomes. PLoS Genet

Hall B, DeRego T, Geib SM. 2014. GAG: the genome annotation generator (version 1.0).

Han MV, Thomas GW, Lugo-Martinez J, Hahn MW. 2013. Estimating gene gain and loss rates in the presence of error in genome assembly and annotation using CAFE 3. Mol Biol Evol 30(8):1987-1997.

Hanson MA, Lemaitre B, Unckless RL. 2019. Dynamic evolution of antimicrobial peptides underscores trade-offs between immunity and ecological fitness. Front Immunol 10:2620.

Harper KE, Bagley RK, Thompson KL, Linnen CR. 2016. Complementary sex determination, inbreeding depression and inbreeding avoidance in a gregarious sawfly. Heredity 117(5):326-335.

Helvig C, Koener JF, Unnithan GC, Feyereisen R. 2004. CYP15A1, the cytochrome P450 that catalyzes epoxidation of methyl farnesoate to juvenile hormone III in cockroach corpora allata. Proc Natl Acad Sci USA 101(12):4024-4029.

Herrig DK, Vertacnik KL, Linnen CR. 2019. Testing the adaptive decoupling hypothesis in a hypermetamorphic and sexually dimorphic insect. bioRxiv 2019.12.20.882803. 
Himejima M, Hobson KR, Otsuka T, Wood DL, Kubo I. 1992. Antimicrobial terpenes from oleoresin of ponderosa pine tree Pinus ponderosa: A defense mechanism against microbial invasion. J Chem Ecol 18(10):1809-1818.

Hughes AL, Nei M. 1992. Maintenance of MHC polymorphism. Nature 335:402-403.

1002

Johnson RM, Harpur BA, Dogantzis KA, Amro Z, Berenbaum MR. 2018. Genomic footprint of evolution of eusociality in bees: floral food use and CYPome "blooms". Insectes Soc 65(3):445-454.

Jones P, Binns D, Chang H-Y, Fraser M, Li W, McAnulla C, McWilliam H, et al. 2014. InterProScan 5: genome-scale protein function classification. Bioinformatics 30(9):1236-1240.

Jones WD, Cayirlioglu P, Kadow IG, Vosshall LB. 2007. Two chemosensory receptors together mediate carbon dioxide detection in Drosophila. Nature 445(7123):86-90.

Jurka J, Kapitonov VV, Pavlicek A, Klonowski P, Kohany O, Walichiewicz J. 2005. Repbase Update, a database of eukaryotic repetitive elements. Cytogenet Genome Res 110(1-4):462-467.

Karev GP, Wolf YI, Rzhetsky AY, Berezovskaya FS, Koonin EV. 2002. Birth and death of protein domains: a simple model of evolution explains power law behavior. BMC Evol Biol 2(1):18. genome of the mountain pine beetle, Dendroctonus ponderosae Hopkins, a major forest pest. Genome Biol 14(3):R27.

Larkin MA, Blackshields G, Brown NP, Chenna R, McGettigan PA, McWilliam H, Valentin F, et al. 2007. Clustal W and Clustal X version 2.0. Bioinformatics 23(21):2947-2948.

LeBoeuf AC, Benton R, Keller L. 2013. The molecular basis of social behavior: models, methods and advances. Curr Opin Neurobiol 23(1):3-10. Bacterial uracil modulates Drosophila DUOX-dependent gut immunity via Hedgehog-induced

1022 Lee Y, Moon SJ, Montell C. 2009. Multiple gustatory receptors required for the caffeine response in

1023 Drosophila. Proc Natl Acad Sci USA 106(11):4495-4500.

1024 Li B, Dewey CN. 2011. RSEM: accurate transcript quantification from RNA-Seq data with or without a 1025 reference genome. BMC Bioinform 12(1):323.

1026 Li H, Durbin R. 2009. Fast and accurate short read alignment with Burrows-Wheeler transform.

1027 Bioinformatics 25(14):1754-1760.

1028 Li R, Yu C, Li Y, Lam T-W, Yiu S-M, Kristiansen K, Wang J. 2009. SOAP2: an improved ultrafast tool 1029 for short read alignment. Bioinformatics 25(15):1966-1967. 
Li W, Schuler MA, Berenbaum MR. 2003. Diversification of furanocoumarin-metabolizing cytochrome P450 monooxygenases in two papilionids: specificity and substrate encounter rate. Proc Natl Acad Sci USA 100(suppl 2):14593-14598.

Li X, Schuler MA, Berenbaum MR. 2007. Molecular mechanisms of metabolic resistance to synthetic and natural xenobiotics. Annu Rev Entomol 52:231-253.

Linnen CR, O'Quin CT, Shackleford T, Sears CR, Lindstedt C. 2018. Genetic basis of body color and spotting pattern in redheaded pine sawfly larvae (Neodiprion lecontei). Genetics 209(1):291-305.

Marçais G, Kingsford C. 2011. A fast, lock-free approach for efficient parallel counting of occurrences of k-mers. Bioinformatics 27(6):764-770.

Martin A, Orgogozo V. 2013. The loci of repeated evolution: a catalog of genetic hotspots of phenotypic variation. Evolution 67(5):1235-1250.

Matsuo T, Sugaya S, Yasukawa J, Aigaki T, Fuyama Y. 2007. Odorant-binding proteins OBP57d and OBP57e affect taste perception and host-plant preference in Drosophila sechellia. PLoS Biology 5(5):e118.

Maxwell DE editor. Proceedings of the 10th International Congress of Entomology. 1958 Montreal, Canada.

McBride CS. 2007. Rapid evolution of smell and taste receptor genes during host specialization in Drosophila sechellia. Proc Natl Acad Sci USA 104(12):4996-5001.

McBride CS, Arguello RJ. 2007. Five Drosophila genomes reveal nonneutral evolution and the signature of host specialization in the chemoreceptor superfamily. Genetics 177(3):1395-1416. of the Asian longhorned beetle (Anoplophora glabripennis), a globally significant invasive species, reveals key functional and evolutionary innovations at the beetle-plant interface. Genome Biol 17:227

McKenzie SK, Fetter-Pruneda I, Ruta V, Kronauer DJC. 2016. Transcriptomics and neuroanatomy of the clonal raider ant implicate an expanded clade of odorant receptors in chemical communication. Proc Natl Acad Sci USA 113(49):14091-14096.

Mittapelly P, Bansal R, Michel A. 2019. Differential expression of cytochrome P450 CYP6 genes in the brown marmorated stink bug, Halyomorpha halys (Hemiptera: Pentatomidae). J Econ Entomol 112(3):1403-1410.

Miyamoto T, Slone J, Song X, Amrein H. 2012. A fructose receptor functions as a nutrient sensor in the Drosophila brain. Cell 151(5):1113-1125.

Moon SJ, Köttgen M, Jiao Y, Xu H, Montell C. 2006. A taste receptor required for the caffeine response in vivo. Curr Biol 16(18):1812-1817. 
1064

1065

1066

1067

1068

1069

1070

1071

1072

1073

1074

1075

1076

1077

1078

1079

1080

1081

1082

1083

1084

1085

1086

1087

1088

1089

1090

1091

1092

1093

1094

1095

Moon SJ, Lee Y, Jiao Y, Montell C. 2009. A Drosophila gustatory receptor essential for aversive taste and inhibiting male-to-male courtship. Curr Biol 19(19):1623-1627.

Mumm R, Hilker M. 2006. Direct and indirect chemical defence of pine against folivorous insects. Trends Plant Sci 11(7):351-358.

Nei M. 2007. The new mutation theory of phenotypic evolution. Proc Natl Acad Sci USA 104(30):1223512242.

Nei M, Rooney AP. 2005. Concerted and birth-and-death evolution of multigene families. Annu Rev Genet 39:121-152.

Niehuis O, Gibson JD, Rosenberg MS, Pannebakker BA, Koevoets T, Judson AK, Desjardins CA, et al. 2010. Recombination and its impact on the genome of the haplodiploid parasitoid wasp Nasonia. PLoS One 5(1):e8597.

Nielsen R, Williamson S, Kim Y, Hubisz MJ, Clark AG, Bustamante C. 2005. Genomic scans for selective sweeps using SNP data. Genome Res 15(11):1566-1575.

Nozawa M, Kawahara Y, Nei M. 2007. Genomic drift and copy number variation of sensory receptor genes in humans. Proc Natl Acad Sci USA 104(51):20421-20426.

Ohno S. 1970. The enormous diversity in genome sizes of fish as a reflection of nature's extensive experiments with gene duplication. Trans Am Fish Soc 99(1):120-130.

Parra G, Bradnam K, Korf I. 2007. CEGMA: a pipeline to accurately annotate core genes in eukaryotic genomes. Bioinformatics 23(9):1061-1067.

Perry GH, Dominy NJ, Claw KG, Lee AS, Fiegler H, Redon R, Werner J, et al. 2007. Diet and the evolution of human amylase gene copy number variation. Nat Genet 39(10):1256-1260.

Peters RS, Krogmann L, Mayer C, Donath A, Gunkel S, Meusemann K, Kozlov A, et al. 2017. Evolutionary history of the Hymenoptera. Curr Biol 27(7):1013-1018.

Petrov DA, Hartl DL. 1997. Trash DNA is what gets thrown away: high rate of DNA loss in Drosophila. Gene 205(1-2):279-289.

Petrov DA, Hartl DL. 1998. High rate of DNA loss in the Drosophila melanogaster and Drosophila virilis species groups. Mol Biol Evol 15(3):293-302.

Petrov DA, Lozovskaya ER, Hartl DL. 1996. High intrinsic rate of DNA loss in Drosophila. Nature 384(6607):346-349.

Poelchau M, Childers C, Moore G, Tsavatapalli V, Evans J, Lee C-Y, Lin H, Lin J-W, Hacket K. 2015. The i5k Workspace@ NAL_enabling genomic data access, visualization and curation of arthropod genomes. Nucleic Acids Res 43(D1):D714-D719. 
Protas ME, Hersey C, Kochanek D, Zhou Y, Wilkens H, Jeffery WR, Zon LI, Borowsky R, Tabin CJ. 2006. Genetic analysis of cavefish reveals molecular convergence in the evolution of albinism. Nat Genet 38(1):107-111.

Qiu Y, Tittiger C, Wicker-Thomas C, Le Goff G, Young S, Wajnberg E, Fricaux T, Taquet N, Blomquist GJ, Feyereisen R. 2012. An insect-specific P450 oxidative decarbonylase for cuticular hydrocarbon biosynthesis. Proc Natl Acad Sci USA 109(37):14858-14863.

1102 Qu Z, Kenny NJ, Lam HM, Chan TF, Chu KH, Bendena WG, Tobe SS, Hui JHL. 2015. How did

1103 arthropod sesquiterpenoids and ecdysteroids arise? Comparison of hormonal pathway genes in noninsect arthropod genomes. Genome Biol Evol 7(7):1951-1959.

Quinlan AR, Hall IM. 2010. BEDTools: a flexible suite of utilities for comparing genomic features. Bioinformatics 26(6):841-842.

R-Core-Team. 2018. R: A language and environment for statistical computing. Vienna, Austria: R Foundation for Statistical Computing.

Ratzka C, Förster F, Liang C, Kupper M, Dandekar T, Feldhaar H, Gross R. 2012. Molecular characterization of antimicrobial peptide genes of the carpenter ant Camponotus floridanus. PLoS One 7(8):e43036.

Rewitz KF, O'Connor MB, Gilbert LI. 2007. Molecular evolution of the insect Halloween family of cytochrome P450s: phylogeny, gene organization and functional conservation. Insect Biochem Mol

1115 Rizzon C, Ponger L, Gaut BS. 2006. Striking similarities in the genomic distribution of tandemly arrayed genes in Arabidopsis and rice. PLoS Comput Biol 2(9):e115.

1117 Robertson HM, Gadau J, Wanner KW. 2010. The insect chemoreceptor superfamily of the parasitoid 1118 jewel wasp Nasonia vitripennis. Insect Mol Biol 19:121-136.

1119 Robertson HM, Kent LB. 2009. Evolution of the gene lineage encoding the carbon dioxide receptor in 1120 insects. J Insect Sci 9(1):19.

1121 Robertson HM, Wanner KW. 2006. The chemoreceptor superfamily in the honey bee, Apis mellifera:

1122 expansion of the odorant, but not gustatory, receptor family. Genome Res 16(11):1395-1403.

1123 Robertson HM, Waterhouse RM, Walden KKO, Ruzzante L, Reijnders MJMF, Coates BS, Legeai F, et 1124 al. 2018. Genome sequence of the wheat stem sawfly, Cephus cinctus, representing an early1125 branching lineage of the Hymenoptera, illuminates evolution of hymenopteran chemoreceptors.

1126 Genome Biol Evol 10(11):2997-3011.

1127 Ross L, Gardner A, Hardy N, West SA. 2013. Ecology, not the genetics of sex determination, determines 1128 who helps in eusocial populations. Curr Biol 23(23):2383-2387. 
Roux J, Privman E, Moretti S, Daub JT, Robinson-Rechavi M, Keller L. 2014. Patterns of positive selection in seven ant genomes. Mol Biol Evol 31(7):1661-1685.

Sadd BM, Barribeau SM, Bloch G, de Graaf DC, Dearden P, Elsik CG, Gadau J, et al. 2015. The genomes of two key bumblebee species with primitive eusocial organization. Genome Biol 16(1):1-

$1133 \quad 32$.

1134 Schindelin J, Arganda-Carreras I, Frise E, Kaynig V, Longair M, Pietzsch T, Preibisch S, et al. 2012. Fiji:

1135 an open-source platform for biological-image analysis. Nature Methods 9(7):676-682.

1136 Sezutsu H, Le Goff G, Feyereisen R. 2013. Origins of P450 diversity. Philos Trans R Soc Lond B Biol $1137 \quad$ Sci 368:20120428.

1138 Simão FA, Waterhouse RM, Ioannidis P, Kriventseva EV, Zdobnov EM. 2015. BUSCO: assessing genome assembly and annotation completeness with single-copy orthologs. Bioinformatics 31(19):3210-3212.

Simone M, Evans JD, Spivak M. 2009. Resin collection and social immunity in honey bees. Evolution 63(11):3016-3022.

Singaravelan N, Nee'man G, Inbar M, Izhaki I. 2005. Feeding responses of free-flying honeybees to secondary compounds mimicking floral nectars. J Chem Ecol 31(12):2791-2804.

1146 Smadja C, Shi P, Butlin RK, Robertson HM. 2009. Large gene family expansions and adaptive evolution

1147 for odorant and gustatory receptors in the pea aphid, Acyrthosiphon pisum. Mol Biol Evol

1148 26(9):2073-2086.

1149 Smit AFA, Hubley R. 2008-2015. RepeatModeler Open-1.0.

1150 Smit AFA, Hubley R, Green P. 2013-2015. RepeatMasker Open-4.0.

1151 Smith CR, Smith CD, Robertson HM, Helmkampf M, Zimin A, Yandell M, Holt C, et al. 2011. Draft

1152 genome of the red harvester ant Pogonomyrmex barbatus. Proc Natl Acad Sci USA 108(14):5667-

$1153 \quad 5672$.

1154 Smith SG. 1941. A new form of spruce sawfly identified by means of its cytology and parthenogenesis. J

1155 Agric Sci 21(5):245-305.

1156 Sohi SS, Ennis TJ. 1981. Chromosomal characterization of cell lines of Neodiprion lecontei

1157 (Hymenoptera: Diprionidae). Proc Entomol Soc Ont 112:45-48.

1158 Souvorov A, Kapustin Y, Kiryutin B, Chetvernin V, Tatusova T, Lipman DJ. 2010. Gnomon-NCBI

1159 eukaryotic gene prediction tool. National Center for Biotechnology Information.

1160 Stamatakis A. 2014. RAxML version 8: a tool for phylogenetic analysis and post-analysis of large 1161 phylogenies. Bioinformatics 30(9):1312-1313. 
Suzuki HC, Ozaki K, Makino T, Uchiyama H, Yajima S, Kawata M. 2018. Evolution of gustatory receptor gene family provides insights into adaptation to diverse host plants in nymphalid butterflies. Genome Biol Evol 10(6):1351-1362.

Thomas JH. 2006. Analysis of homologous gene clusters in Caenorhabditis elegans reveals striking regional cluster domains. Genetics 172(1):127-143.

1167 Tian C, Gao B, Fang Q, Ye G, Zhu S. 2010. Antimicrobial peptide-like genes in Nasonia vitripennis: a 1168 genomic perspective. BMC Genom 11(1):187.

1169 Trapp S, Croteau R. 2001. Defensive resin biosynthesis in conifers. Annu Rev Plant Biol 52(1):689-724.

1170 Vieira FG, Sánchez-Gracia A, Rozas J. 2007. Comparative genomic analysis of the odorant-binding protein family in 12 Drosophila genomes: purifying selection and birth-and-death evolution. Genome Biol 8(11):R235.

Vitti JJ, Grossman SR, Sabeti PC. 2013. Detecting natural selection in genomic data. Annu Rev Genet 4797-120.

1175 Vosshall LB, Wong AM, Axel R. 2000. An olfactory sensory map in the fly brain. Cell 102(2):147-159.

1176 Wanner KW, Robertson HM. 2008. The gustatory receptor family in the silkworm moth Bombyx mori is

1177 characterized by a large expansion of a single lineage of putative bitter receptors. Insect Mol Biol 17(6):621-629.

Wiens JJ, Lapoint RT, Whiteman NK. 2015. Herbivory increases diversification across insect clades. Nat $1180 \quad$ Commun 6(1):1-7.

1181 Wilson LF, Wilkinson RC, Averill RC. 1992. Redheaded pine sawfly: its ecology and management. US

1182 Department of Agriculture, Forest Service, editor. Agriculture Handbook No. 694. Washington, DC.

1183 Xu Y-L, He P, Zhang L, Fang S-Q, Dong S-L, Zhang Y-J, Li F. 2009. Large-scale identification of

1184 odorant-binding proteins and chemosensory proteins from expressed sequence tags in insects. BMC

1185 Genom 10(1):632.

1186 Yang J, Chen X, Bai J, Fang D, Qiu Y, Jiang W, Yuan H, et al. 2016. The Sinocyclocheilus cavefish

1187 genome provides insights into cave adaptation. BMC Biol 14(1):1-13.

1188 Yang Z. 2007. PAML 4: phylogenetic analysis by maximum likelihood. Mol Biol Evol 24(8):1586-1591.

1189 Zhang J. 2003. Evolution by gene duplication: an update. Trends Ecol Evol 18(6):292-298.

1190 Zhang L, Gaut BS. 2003. Does recombination shape the distribution and evolution of tandemly arrayed

1191 genes (TAGs) in the Arabidopsis thaliana genome? Genome Res 13(12):2533-2540.

1192 Zhang Z, Zhu S. 2012. Comparative genomics analysis of five families of antimicrobial peptide-like

1193 genes in seven ant species. Dev Comp Immunol 38(2):262-274.

1194 Zhen Y, Aardema ML, Medina EM, Schumer M, Andolfatto P. 2012. Parallel molecular evolution in an 1195 herbivore community. Science 337(6102):1634-1637. 
Zhou X, Rokas A, Berger SL, Liebig J, Ray A, Zwiebel LJ. 2015. Chemoreceptor evolution in hymenoptera and its implications for the evolution of eusociality. Genome Biol Evol 7(8):2407-2416.

\section{Figure Legends}

1200 Figure 1. Like other diprionids, N. lecontei has multiple morphological and behavioral adaptations

1201 to Pinus foliage. A. An egg-laying N. lecontei female demonstrating several adaptations for dealing with

1202 thick, resinous pine needles, including: a robust saw-like ovipositor (visible within the needle), a tendency

1203 to lay many closely spaced eggs per needles, and a tendency to cut resin-draining slits on egg-bearing

1204 needles (circled). B. Prior to hatching, N. lecontei eggs absorb water from the host, causing the eggs to

1205 swell and the pockets to open. Throughout development, embryos are in close contact with living host

1206 tissue. C. Early-instar larvae have skeletonizing feeding behavior in which only the outer needle tissue is

1207 consumed, leaving the resinous interior intact. This strategy prevents small larvae from being

1208 overwhelmed by sticky resin. D. Mid- and late-instar larvae consume the entire pine needle. Larvae

1209 sequester pine resin in specialized pouches for use in self-defense (All photos by R.K. Bagley).

Figure 2. Optical sections through the antennal lobes of adult female (left) and male (right) $N$.

1212 lecontei. White arrows indicate regions of male-specific synaptic clusters. Scale bars $=500 \mu \mathrm{m}$. anchored assembly described in Linnen et al. 2018 (GenBank accession numbers are as follows: LG1 = CM009916.1; LG2 = CM009917.1; LG3 = CM009918.1; LG4 = CM009919.1; LG5 = CM009920.1; LG6 = CM009921.1; LG7 = CM009922.1). Gene family abbreviations: OR (olfactory receptor), GR (gustatory receptor), OBP (odorant binding protein), CYP (cytochrome P450), AMP (antimicrobial protein). Each gene family is represented by a different color. Horizontal lines indicate the approximate locations of genes within LG; diagonal lines that connect to horizontal lines are used to highlight groups of genes that met our clustering criteria. Genes that were found on scaffolds that have not been placed on linkage groups are indicated on the bottom left, with abbreviated scaffold names given in parentheses (e.g., S-210 = scaffold_210 = LGIB01000210.1 in the assemblies available on NCBI). 
1231 Figure 5. Ecological correlates of gene family size in Hymenoptera. Each point represents the number

1232 of intact genes for a hymenopteran species for which both manually curated gene annotations and

1233 ecological data are available. Asterisks indicate that gene number varies significantly among the

1234 ecological categories under consideration; for significant categories with $>2$ groups, letters indicate

1235 significance in post-hoc tests (groups that do not share a letter are significantly different). Gene number

1236 and ecological data for all taxa are provided in Table S8. 
Table 1. Summary of within-genome signatures of adaptive expansions and contractions

1238 for five environmentally responsive gene families.

\begin{tabular}{|c|c|c|c|}
\hline & Gene family size & $\begin{array}{c}\text { Genomic } \\
\text { Clustering }\end{array}$ & Molecular evolution \\
\hline
\end{tabular}

\begin{tabular}{|l|ccccc|cc|ccc|}
\multicolumn{1}{|c|}{ Gene family $^{*}$} & $\begin{array}{c}\text { Intact } \\
\text { genes }\end{array}$ & Partial & Pseudo & $\begin{array}{c}\text { Total } \\
\text { genes }\end{array}$ & $\begin{array}{c}\text { Prop. } \\
\text { pseudo }\end{array}$ & $\begin{array}{c}\text { Prop. in } \\
\text { clusters }^{\dagger}\end{array}$ & $\begin{array}{c}\text { Largest } \\
\text { cluster }\end{array}$ & $\begin{array}{c}\text { Neodiprion- } \\
\text { specific } \\
\text { clades }^{\ddagger}\end{array}$ & $\begin{array}{c}\text { Significant } \\
\text { branch }_{\text {tests }^{\S}}\end{array}$ & $\begin{array}{c}\text { Significant } \\
\text { site tests }^{* *}\end{array}$ \\
\hline OR & 52 & 3 & 1 & 56 & 0.02 & 0.59 & 8 & 3 & 1 & 0 \\
GR & 41 & 2 & 2 & $44^{\dagger \dagger}$ & 0.05 & 0.76 & 10 & 3 & 1 & 1 \\
OBP & 13 & 0 & 0 & 13 & 0 & 0.38 & 3 & 0 & $\mathrm{n} / \mathrm{a}$ & $\mathrm{n} / \mathrm{a}$ \\
CYP (all) & 93 & 2 & 12 & 107 & 0.11 & 0.66 & 16 & 5 & 2 & 0 \\
CYP2 clan & 9 & 0 & 0 & 9 & 0 & 0.33 & 2 & 0 & 0 & 0 \\
CYP3 clan & 47 & 0 & 8 & 55 & 0.15 & 0.81 & 16 & 4 & 2 & 0 \\
$\quad$ CYP4 clan & 27 & 2 & 4 & 33 & 0.12 & 0.55 & 3 & 1 & 0 & 0 \\
mito CYP clan & 10 & 0 & 0 & 10 & 0 & 0.50 & 3 & 0 & 0 & 0 \\
AMP & 21 & 0 & 0 & 21 & 0 & 0.95 & 15 & $?$ & 0 & 0 \\
\hline
\end{tabular}

\footnotetext{
* Abbreviations: $\mathrm{OR}=$ olfactory receptor genes; GR = gustatory receptor genes; $\mathrm{OBP}=$ odorant binding protein genes; CYP = cytochrome P450 genes ("clans" refer to four major clades of CYPs present in insects); AMP = antimicrobial peptide genes.

${ }^{\dagger}$ Calculated as: (number of genes in clusters of 2 or more)/(genes for which clustering could be evaluated).

$¥$ Defined as monophyletic clusters of 5 or more Neodiprion paralogs with a bootstrap support $\geq$ $70 \%$ in an amino acid phylogeny constructed with gene annotations from Neodiprion, select Hymenoptera, and Drosophila melanogaster.

$\S$ To be counted, clades had to reject both 1-ratio and fixed-ratio models in $\mathrm{dN} / \mathrm{dS}$ branch tests (see Table 2).

${ }^{* *}$ To be counted, clades had to reject both M7 and M8a models in dN/dS site tests (see Table 2).

${ }^{\dagger}$ One gene was both a partial annotation and a pseudogene.

tH Low bootstrap support precluded the identification of Neodiprion-specific clades.
} 
Table 2. Likelihood-ratio tests (LRTs) of positive selection on Neodiprion-specific clades (branch models) and on amino acid sites within these clades (site models).

\begin{tabular}{|c|c|c|c|c|c|}
\hline Clade Names* & $\mathrm{n}^{\dagger}$ & Model comparison & $\begin{array}{c}\text { LRT } \\
\text { statistic }^{\S}\end{array}$ & $\mathrm{df}$ & P-value ** \\
\hline \multicolumn{6}{|c|}{ Olfactory Receptor } \\
\hline \multirow{4}{*}{ Clade 1} & \multirow{4}{*}{6} & M8 vs M7 & 2.932 & 2 & 0.231 \\
\hline & & M8 vs M8a & 0.748 & 1 & 0.387 \\
\hline & & 2 ratio vs 1 ratio & 5.408 & 1 & 0.020 \\
\hline & & 2 ratio vs neutral & 7.800 & 1 & 0.005 \\
\hline \multirow{4}{*}{ Clade 2} & \multirow{4}{*}{5} & M8 vs M7 & 3.941 & 2 & 0.139 \\
\hline & & M8 vs M8a & 1.525 & 1 & 0.217 \\
\hline & & 2 ratio vs 1 ratio & 1.426 & 1 & 0.232 \\
\hline & & 2 ratio vs neutral & 0.050 & 1 & 0.822 \\
\hline \multirow{4}{*}{ Clade 3} & \multirow{4}{*}{5} & M8 vs M7 & 0 & 2 & 1 \\
\hline & & M8 vs M8a & 0 & 1 & 1 \\
\hline & & 2 ratio vs 1 ratio & 2.395 & 1 & 0.122 \\
\hline & & 2 ratio vs neutral & 6.371 & 1 & 0.012 \\
\hline \multicolumn{6}{|c|}{ Gustatory Receptor } \\
\hline \multirow{4}{*}{ Clade 1} & \multirow{4}{*}{7} & M8 vs M7 & 0.809 & 2 & 0.667 \\
\hline & & M8 vs M8a & 0.379 & 1 & 0.538 \\
\hline & & 2 ratio vs 1 ratio & 0.003 & 1 & 0.954 \\
\hline & & 2 ratio vs neutral & 0.645 & 1 & 0.422 \\
\hline \multirow{4}{*}{ Clade 2} & \multirow{4}{*}{8} & M8 vs M7 & 6.049 & 2 & 0.049 \\
\hline & & M8 vs M8a & 2.654 & 1 & 0.103 \\
\hline & & 2 ratio vs 1 ratio & 0.003 & 1 & 0.959 \\
\hline & & 2 ratio vs neutral & 0.781 & 1 & 0.377 \\
\hline \multirow{4}{*}{ Clade 3} & \multirow{4}{*}{5} & M8 vs M7 & 39.328 & 2 & $2.884 \mathrm{e}-09$ \\
\hline & & M8 vs M8a & 35.167 & 1 & $3.026 \mathrm{e}-09$ \\
\hline & & 2 ratio vs 1 ratio & 14.789 & 1 & $1.202 \mathrm{e}-04$ \\
\hline & & 2 ratio vs 2 ratio neutral & 27.810 & 1 & $1.338 \mathrm{e}-07$ \\
\hline \multicolumn{6}{|c|}{ Cytochrome P450 } \\
\hline \multirow{4}{*}{$\begin{array}{c}\text { Clade } 1 \\
\text { (CYP4 clan) }\end{array}$} & \multirow{4}{*}{8} & M8 vs M7 & 0.615 & 2 & 0.735 \\
\hline & & M8 vs M8a & 0.866 & 1 & 0.352 \\
\hline & & 2 ratio vs 1 ratio & 0.658 & 1 & 0.417 \\
\hline & & 2 ratio vs 2 ratio neutral & 0.089 & 1 & 0.766 \\
\hline \multirow{3}{*}{$\begin{array}{c}\text { Clade } 2 \\
\text { (CYP3 clan) }\end{array}$} & \multirow{3}{*}{19} & M8 vs M7 & 0 & 2 & 1 \\
\hline & & M8 vs M8a & 0 & 1 & 1 \\
\hline & & 2 ratio vs 1 ratio & 2.077 & 1 & 0.149 \\
\hline
\end{tabular}

${ }^{*}$ Clade names are as in Figures S1a,S1b,S2a,S2b,S3a,S3b,S4a,S4b, and S5a.

$\dagger$ Putatively functional genes. Pseudogenes and partial annotations were excluded from analysis.

* Site models unshaded; neutral M7 and M8a do not allow for positive selection. Branch models shaded; 1 ratio estimates a single $\omega$ value for all branches, 2 ratio estimates a separate $\omega$ value for the foreground branch, 2 ratio neutral fixes $\omega=1$ for all branches.

${ }^{\S}$ Likelihood ratio test statistic, calculated twice the difference in model log likelihoods.

${ }^{* *}$ Bolded values are significant at critical value 0.05 . 


\begin{tabular}{|c|c|c|c|c|c|}
\hline & & 2 ratio vs 2 ratio neutral & 0.076 & 1 & 0.783 \\
\hline \multirow{4}{*}{$\begin{array}{c}\text { Clade } 3 \\
\text { (CYP3 clan) }\end{array}$} & \multirow{4}{*}{6} & M8 vs M7 & 7.152 & 2 & 0.028 \\
\hline & & M8 vs M8a & 0.649 & 1 & 0.421 \\
\hline & & 2 ratio vs 1 ratio & 6.325 & 1 & 0.012 \\
\hline & & 2 ratio vs 2 ratio neutral & 14.261 & 1 & 1.59 e- 04 \\
\hline \multirow{4}{*}{$\begin{array}{c}\text { Clade } 4 \\
\text { (CYP3 clan) }\end{array}$} & \multirow{4}{*}{6} & M8 vs M7 & 0 & 2 & 1 \\
\hline & & M8 vs M8a & 0.151 & 1 & 0.697 \\
\hline & & 2 ratio vs 1 ratio & 0.002 & 1 & 0.964 \\
\hline & & 2 ratio vs 2 ratio neutral & 0.936 & 1 & 0.333 \\
\hline \multirow{4}{*}{$\begin{array}{c}\text { Clade } 5 \\
\text { (CYP3 clan) }\end{array}$} & \multirow{4}{*}{5} & M8 vs M7 & 0 & 2 & 1 \\
\hline & & M8 vs M8a & 0 & 1 & 1 \\
\hline & & 2 ratio vs 1 ratio & 5.327 & 1 & 0.021 \\
\hline & & 2 ratio vs 2 ratio neutral & 12.286 & 1 & $4.56 \mathrm{e}-04$ \\
\hline \multicolumn{6}{|c|}{ Hisnavicin (Antimicrobial Peptide) } \\
\hline \multirow{4}{*}{ Clade $1^{\dagger \dagger}$} & \multirow{4}{*}{15} & M8 vs M7 & 2.388 & 2 & 0.665 \\
\hline & & M8 vs M8a & 0 & 1 & 1 \\
\hline & & 2 ratio vs 1 ratio & 7.908 & 1 & 0.010 \\
\hline & & 2 ratio vs 2 ratio neutral & 0.999 & 1 & 0.635 \\
\hline
\end{tabular}

${ }^{+\dagger}$ Although this clade did not meet the bootstrap criteria for species-specific clades $(>70)$, it was included in this analysis because it contained almost all $N$. lecontei hisnavicin paralogs. 

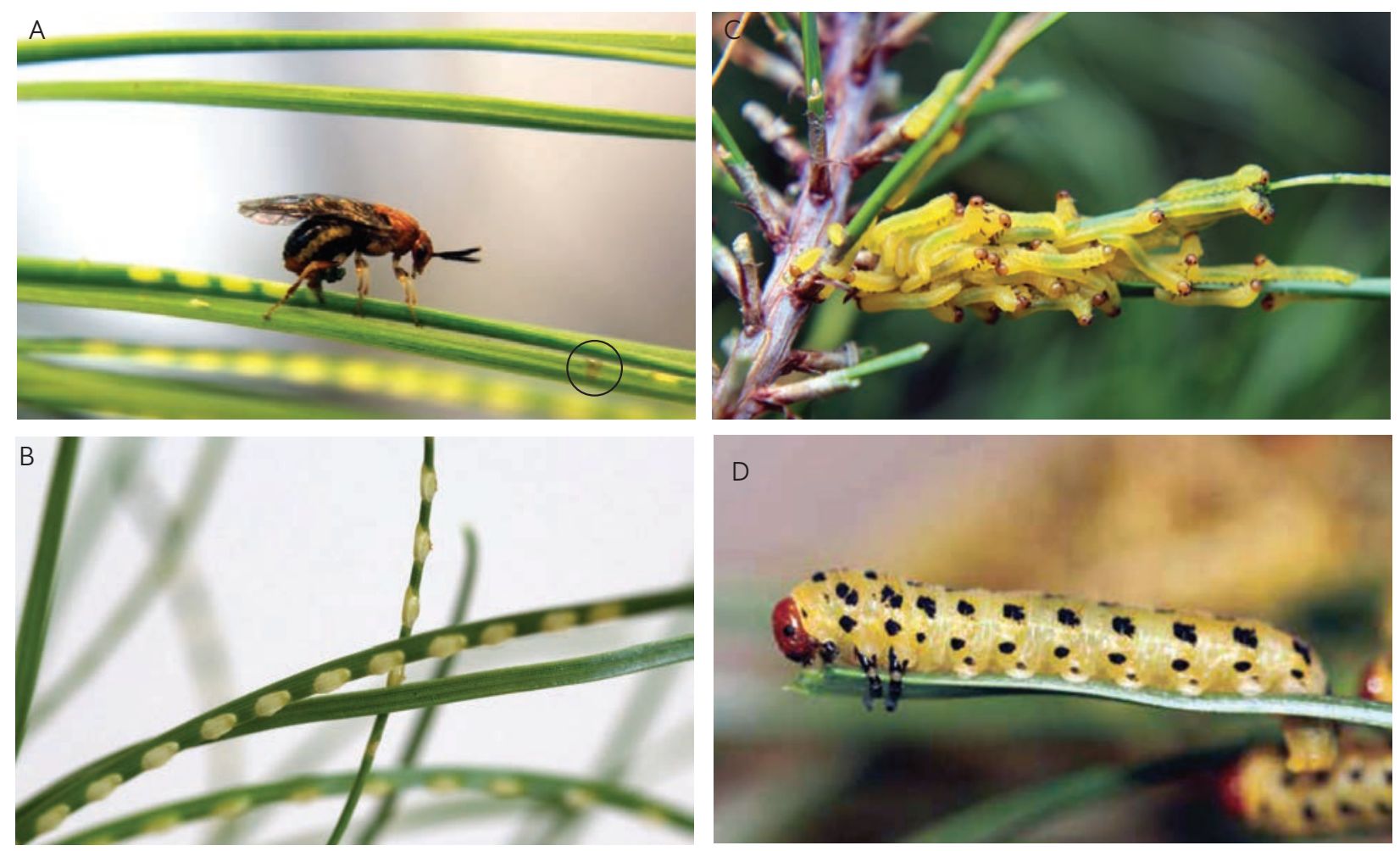

Figure 1. Like other diprionids, $N$. lecontei has multiple morphological and behavioral adaptations to Pinus foliage. A. An egg-laying $N$. lecontei female demonstrating several adaptations for dealing with thick, resinous pine needles, including: a robust saw-like ovipositor (visible within the needle), a tendency to lay many closely spaced eggs per needles, and a tendency to cut resin-draining slits on egg-bearing needles (circled). B. Prior to hatching, $N$. lecontei eggs absorb water from the host, causing the eggs to swell and the pockets to open. Throughout development, embryos are in close contact with living host tissue. C. Early-instar larvae have skeletonizing feeding behavior in which only the outer needle tissue is consumed, leaving the resinous interior intact. This strategy prevents small larvae from being overwhelmed by sticky resin. D. Mid- and late-instar larvae consume the entire pine needle. Larvae sequester pine resin in specialized pouches for use in self-defense (All photos by R.K. Bagley). 

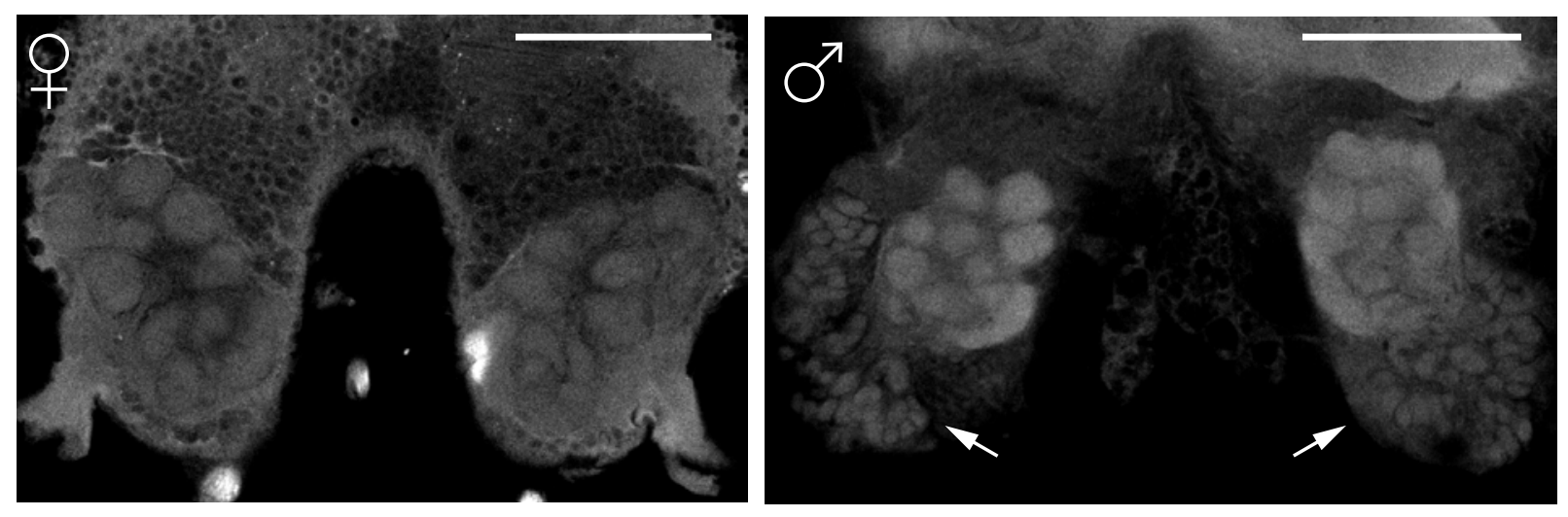

Figure 2. Optical sections through the antennal lobes of adult female (left) and male (right) N. lecontei. White arrows indicate regions of male-specific synaptic clusters. Scale bars $=500$ $\mu \mathrm{m}$. 

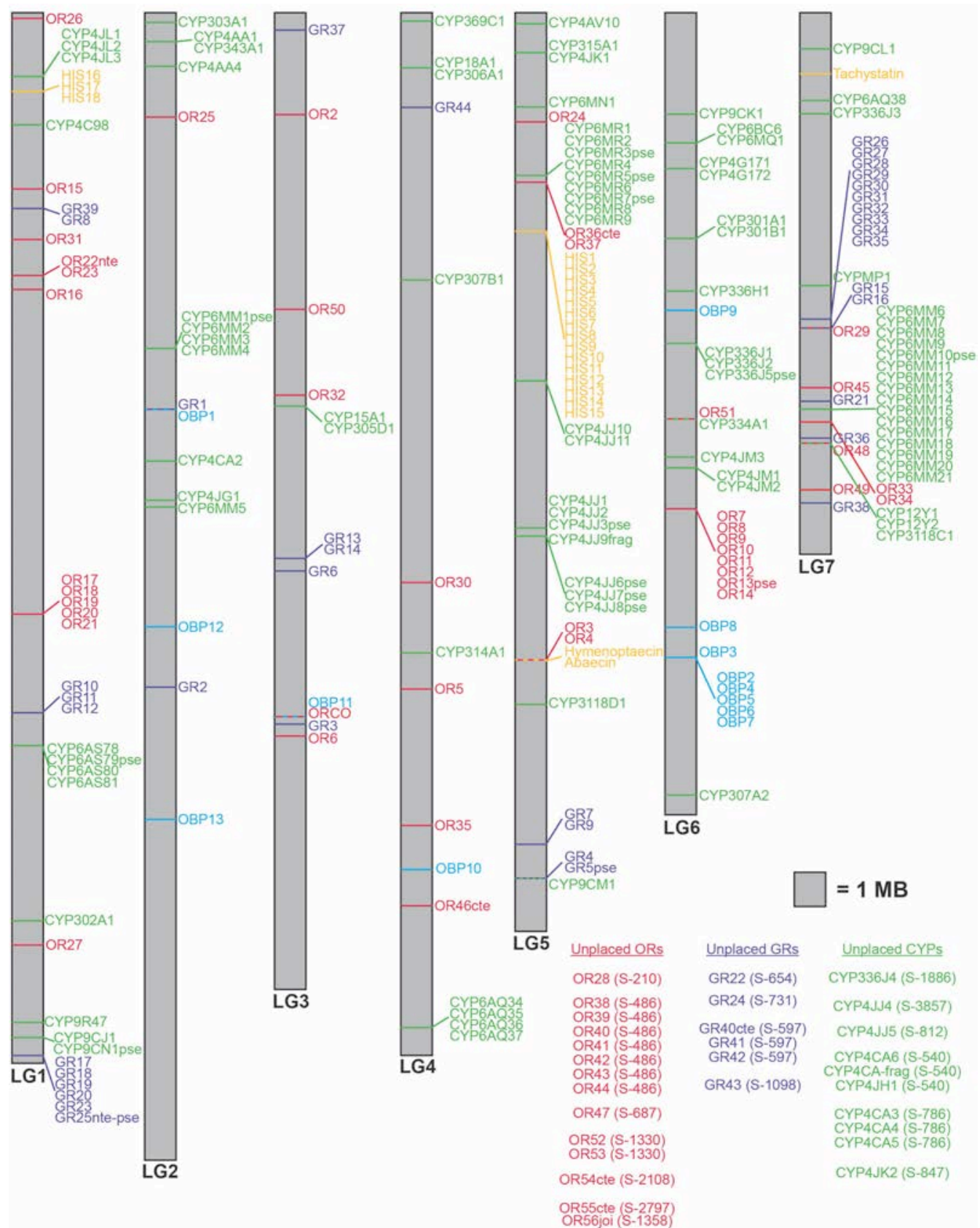

Figure 3. Position of genes belonging to five environmentally responsive gene families along seven $\mathrm{N}$. lecontei linkage groups. Linkage groups (LG) are drawn to scale and ordered as in the linkage-group anchored assembly described in Linnen et al. 2018 (GenBank accession numbers are as follows: LG1 = CM009916.1; LG2 = CM009917.1; LG3 = CM009918.1; LG4 = CM009919.1; LG5 = CM009920.1; LG6 = CM009921.1; LG7 = CM009922.1). Gene family abbreviations: OR (olfactory receptor), GR (gustatory receptor), OBP (odorant binding protein), CYP (cytochrome P450), AMP (antimicrobial protein). Each gene family is represented by a different color. Horizontal lines indicate the approximate locations of genes within LG; diagonal lines that connect to horizontal lines are used to highlight groups of genes that met our clustering criteria. Genes that were found on scaffolds that have not been placed on linkage groups are indicated on the bottom left, with abbreviated scaffold names given in parentheses (e.g., S-210 = scaffold_210 = LGIB01000210.1 in the assemblies available on NCBI). 


\begin{tabular}{|c|c|c|c|c|c|c|}
\hline & & OR & GR & OBP & $\mathrm{P} 450$ & AMP \\
\hline \multirow{13}{*}{ Bee } & Bombus terrestris & 151 & 21 & 16 & 44 & \\
\hline & Melipona quadrifasciata & 142 & 10 & 6 & & \\
\hline & \& Apis mellifera & 169 & 10 & 21 & 46 & 6 \\
\hline & Apis cerana & 119 & 10 & & 41 & \\
\hline & Apis dorsata & & & & 42 & \\
\hline & - Apis florea & 159 & & & 44 & \\
\hline & - Habropoda laboriosa & 100 & & & 38 & \\
\hline & [ Euglossa dilemma & 123 & 13 & 15 & & \\
\hline & Eufriesea mexicana & 111 & 16 & 13 & 45 & \\
\hline & - Megachile rotundata & & & & 49 & \\
\hline & Osmia bicornis bicornis & & & & 47 & \\
\hline & - Lasioglossum albipes & 158 & 23 & & & \\
\hline & Dufourea novaeangliae & 77 & & & 45 & \\
\hline \multirow{10}{*}{ Ant } & [ Solenopsis invicta & 333 & 219 & 18 & & 7 \\
\hline & [ Cardiocondyla obscurior & 232 & 34 & & & \\
\hline & Monomorium pharaonis & 240 & 159 & & & \\
\hline & L Pogonomyrmex barbatus & 274 & 58 & 16 & 72 & 10 \\
\hline & - Acromyrmex echinatior & 375 & 116 & & 73 & \\
\hline & Atta cephalotes & 341 & 89 & & 52 & \\
\hline & - Camponotus floridanus & 352 & 46 & 13 & 128 & 6 \\
\hline & Linepithema humile & 301 & 93 & 13 & 111 & 6 \\
\hline & Cerapachys biroi & 256 & 20 & 15 & 69 & 6 \\
\hline & - Harpegnathos saltator & 347 & 17 & 13 & 95 & 8 \\
\hline \multirow{3}{*}{ Wasp } & Nasonia vitripennis & 217 & 47 & 82 & 92 & 44 \\
\hline & Ceratosolen solmsi & 56 & 5 & 7 & 34 & 8 \\
\hline & L Microplitis demolitor & 203 & 79 & & & \\
\hline Sawfly & Neodiprion lecontei & 52 & 41 & 13 & 94 & 21 \\
\hline
\end{tabular}

Figure 4. Number of intact genes in hymenopteran genomes for each of five environmentally responsive gene families. Phylogenetic relationships are as in Moreau et al. (2006); Hedtke et al. (2013); Roux et al. (2014); Brand et al. (2017); Branstetter et al. (2017); Peters et al. (2017). Branch lengths are arbitrary. Gene family abbreviations are as in Figure 3. 

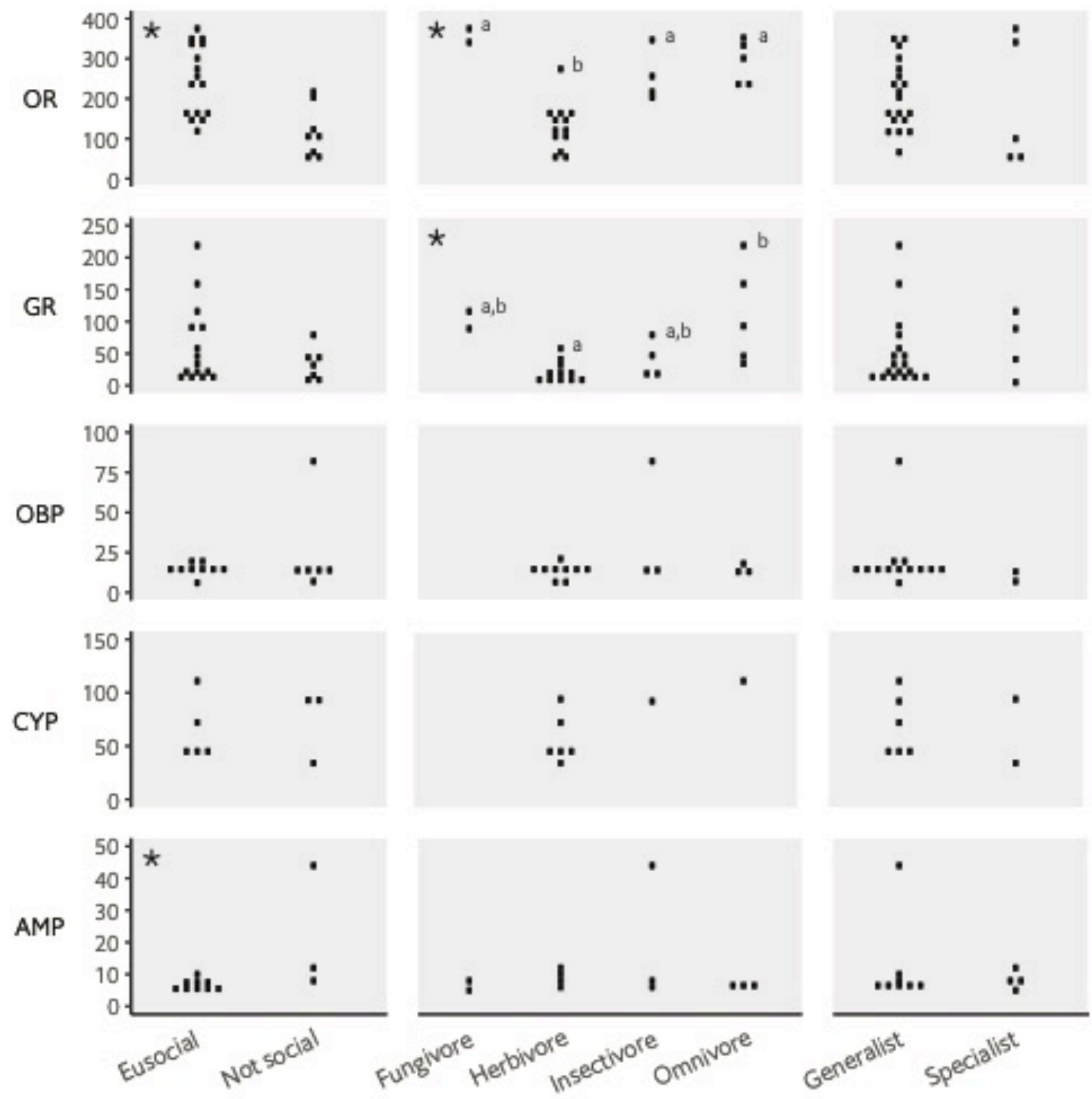

Figure 5. Ecological correlates of gene family size in Hymenoptera. Each point represents the number of intact genes for a hymenopteran species for which both manually curated gene annotations and ecological data are available. Asterisks indicate that gene number varies significantly among the ecological categories under consideration; for significant categories with $>2$ groups, letters indicate significance in post-hoc tests (groups that do not share a letter are significantly different). Gene number and ecological data for all taxa are provided in Table S8. 